\title{
Activation of Chaperon-Mediated Autophagy Via Modulation of Hsp90 Activity Inhibits Tumor Growth Under Hypoxia
}

\author{
Jun-Kyu Byun
}

Kyungpook National University

\section{Sun Hee Lee}

Kyungpook National University

Eui Jung Moon

Oxford University: University of Oxford

Myo-Hyeon Park

Kyungpook National University

Hyeonha Jang

Kyungpook National University

Douglas H. Weitzel

Duke University

Hyun-Hwi Kim

Gachon University

Nikita Basnet

Kyungpook National University

Do-Yeon Kwon

Duke University

Chen-Ting Lee

Duke University

Tesia N. Stephenson

Duke University

Ji-Hak Jeong

Kyungpook National University

Bhargav A. Patel

Notre Dame University

Sung Jean Park

Gachon University

Im Sook Song

Kyungpook National University

Jen-Tsan Chi 
Duke University

\section{Mark W. Dewhirst}

Duke University

Jiyong Hong

Duke University

You Mie Lee ( $\square$ lym@knu.ac.kr)

Kyungpook National University

\section{Research}

Keywords: Chaperon-mediated autophagy, Manassantin A, HIF-1a, Hsp90, F1F0-ATP synthase

Posted Date: October 12th, 2021

DOI: https://doi.org/10.21203/rs.3.rs-958907/v1

License: (c) (i) This work is licensed under a Creative Commons Attribution 4.0 International License. Read Full License 


\section{Abstract}

Background: Chaperon-mediated autophagy (CMA) is a target specific degradation pathway among autophagic processes. Although CMA plays critical roles in tumor progression in general, the role of CMA in tumor progression under hypoxia is poorly understood. We investigated the role of CMA in hypoxic tumor using a novel Hsp90-mediated modulator of CMA.

Methods: We examined whether manassantin A (ManA), known as a potent inhibitor of HIF-1a, is a CMA modulator using biochemical, molecular, and cell biology approaches. We analyzed the effects of ManA on Hsp90 chaperone function by using Significant Analysis of Microarray, luciferase refolding assay, HS10 resin binding assay, NMR spectroscopy, and SPR assay. We investigated tumor growth in response to monotherapy and combination therapy with ManA and anti-programmed death-1 (PD-1) antibody in vivo. To assess the clinical efficacy of CMA-related genes, we analyzed the gene expressions of HIF-1a, HSP90AA1, and transcription factor EB (TFEB) using TCGA datasets. Finally, we assessed in vivo/in vitro absorption, distribution, metabolism, and excretion properties of ManA.

Results: ManA inhibits Hsp90 chaperone function through disruption of the Hsp90/ $\mathrm{F}_{1} \mathrm{~F}_{0}$-ATP synthase (chaperone/co-chaperone) complex. The inhibition of Hsp90 enhances the interaction of CMA substrates and LAMP-2A as well as TFEB nuclear localization, thus leading to CMA activation. Importantly, CMA activation not only retards tumor growth in vitro and in vivo, but also displays cooperative antitumor activity with anti-PD-1 antibody in vivo. An in-depth analysis of TCGA datasets shows that combined expression of HSP90AA $1^{\text {High }} /$ HIF1A ${ }^{\text {High }}$ or TFEB ${ }^{\text {Low }} /$ HIF1A $^{\text {High }}$ is strongly correlated with poor prognosis in lung cancer patients.

Conclusions: ManA-induced inhibition of Hsp90 promotes CMA activity and decreases the stability of CMA substrates such as HIF-1a protein, leading to a marked reduction of hypoxic tumor growth. Therefore, targeting CMA activity via Hsp90 may present a promising therapeutic strategy for hypoxic tumor.

\section{Background}

Autophagy is an adaptive response to external or internal stresses which regulates targeting and degradation of intracellular proteins as well as organelles in the lysosomes [1]. Of the different autophagic pathways that coexist in all cells, chaperon-mediated autophagy (CMA) is significantly different from other types of autophagy. For CMA-mediated protein degradation, substrate proteins having the pentapeptide motif KFERQ bind with the heat shock cognate protein of $70 \mathrm{kDa}$ (Hsc70) chaperon, which form a substrate-chaperone complex. This complex docks at the lysosomal membrane through interaction with lysosomal-associated membrane protein 2A (LAMP-2A), which is a rate-limiting step of CMA pathway [2]. Several studies showed that excessive CMA activation enhances effect of chemotherapy [3] and degrades mutated p53, leading to inhibition of tumor development [4]. Moreover, degradation of Myc [5] or hexokinase II (HKII) [6] through CMA prevents cell transformation and promotes 
metabolic catastrophe in cancer cells, respectively. These reports suggested that activation of CMA could be a promising new treatment strategy for cancer.

Molecular chaperones are essential in maintaining proteostasis and cellular homeostasis under constantly changing environmental conditions. Hsp90 is one of the major molecular chaperones and assists a variety of client proteins to fold properly into their active conformation by consuming energy from ATP hydrolysis [7]. Several hundred proteins are Hsp90 client proteins, which makes Hsp90 a central modulator of important biological processes including DNA repair, development, and immune responses [7]. In addition to its essential roles in cellular homeostasis, increasing evidence suggests that Hsp90 inhibitors regulates the CMA pathway rather than macroautophagy and the proteasome-dependent pathway. To date, however, the exact role of Hsp90 in CMA remains rather unclear in hypoxic tumor. Given that CMA induces the degradation of HIF-1a proteins [8], the CMA pathway may be relevant to altered tumor growth under hypoxic conditions.

Hypoxia, usually defined as $<2 \%$ of $\mathrm{O}_{2}$, occurs in a variety of pathological conditions, including cancer progression, stroke, tissue ischemia, and inflammation. Animal tissues cope with the stress of hypoxia by regulating hypoxia-inducible factor-1a (HIF-1a) [9-11]. HIF-1a is stabilized under hypoxic conditions via inhibition of oxygen-dependent proline hydroxylation, whereas HIF-1a is degraded under normoxic conditions via hydroxylation by proline hydroxylases (PHDs) through the proteasome pathway [12]. Tumors adapt to hypoxia by increasing angiogenesis, altering apoptosis, and regulating metabolism [13], which primarily is to ensure cell survival under short- and long-term hypoxia. The adaptations mediated by HIF-1 a further make tumors both more aggressive and resistant to radiotherapy or chemotherapy [1417], resulting in poor clinical outcomes [18-20]. Pathological and clinical evidence suggests that HIF-1A is a validated, promising drug target for cancer [21, 22]. However, as a tool for treating cancer, HIF-1a inhibition under hypoxic conditions is challenging in that the activation of PHDs to degrade HIF-1a is completely dependent on oxygen availability [12] but tumor microenvironment is still hypoxic due to tumor vascular abnormality [23]. Therefore, other innovative approaches to HIF-1 a degradation under hypoxic conditions need to be developed.

Manassantin A (ManA), a natural product isolated from an aquatic plant Saururus cernuus $\mathrm{L}$. (Saururaceae), is a potent inhibitor of HIF-1a activity. Recently, we reported the structure-activity relationship (SAR) and biological characterization of ManA [24, 25]. However, the precise molecular mechanisms by which ManA inhibits tumor growth and HIF-1a under hypoxia are largely unknown.

In this study, we demonstrated that CMA activation is crucial for inhibition of tumor growth under hypoxic conditions. We established the unique molecular mechanism of ManA on CMA-lysosomal degradation pathway and investigated how CMA modulates the antitumor activity of ManA under hypoxic conditions. Our results show that ManA inhibits Hsp90 function by disrupting the Hsp90/ $\mathrm{F}_{1} \mathrm{~F}_{0}$-ATP synthase (chaperone/co-chaperone) complex. Inhibiting Hsp90 function by ManA increases the interaction of CMA substrates and LAMP2A and the nuclear localization of transcription factor EB (TFEB). The results indicate that increased CMA activity is regulated by inhibition of Hsp90 under hypoxic conditions which 
leads to antitumor efficacy. Therefore, our study suggests the possibility that targeting CMA activity may present a promising therapeutic strategy for hypoxic tumor.

\section{Methods}

\section{Cell culture}

The human embryonic kidney epithelial cell line HEK293T, mouse lung cancer cell line LLC, human lung cancer cell line A549, human liver cancer cell line Huh7, human breast cancer cell lines MDA-MB-231, MCF7, and BT-474 were obtained from American Type Culture Collection (Manassas, VA). ATG7 wild type and knockout HeLa cells were kindly provided by Dr. Masaaki Komatsu in Tokyo Metropolitan Institute of Medical Science. All cell lines except for A549 and MDA-MB-231 were maintained in DMEM medium with $10 \%$ fetal bovine serum (FBS) and 1\% penicillin/streptomycin (P/S). A549 and MDA-MB-231 cell lines were maintained in RPMI 1640 and MEM medium with $10 \%$ FBS and $1 \%$ P/S, respectively. For the hypoxic conditions, hypoxic chambers (ASTEC, Fukuoka, Japan) were used to maintain low oxygen tension $\left(1 \% \mathrm{O}_{2}, 5 \% \mathrm{CO}_{2}\right.$, and balanced with $\left.\mathrm{N}_{2}\right)$.

\section{Chemicals}

All chemicals except for manassantin A, cruentaren A, anti-PD-1 mAb, AR7, and 17-AAG were purchased from Sigma (St. Louis, MO). Manassantin A and cruentaren A were synthesized according to the literatures [26, 27]. Anti-PD-1 mAb were purchased from BioXCell (West Lebanon, NH). AR7 and 17-AAG were from Selleckchem (Houston, TX).

\section{Mice}

Animal experiments were performed using C57BL/6 mice (Japan SLC, Shizuoka, Japan) which were handled in strict compliance with the guidelines for care and use of laboratory animals issued (KNU 2017-145) by the Institutional Ethical Animal Care Committee of Kyungpook National University (Daegu, Korea).

\section{Stable knockdown of LAMP-2A}

Control short hairpin scramble (shScramble) and shLAMP-2A constructs (GeneCopoeia, Rockville, M) were used for stable knockdown of LAMP-2A in LLC cells. Cells were infected using lentiviruses containing shScramble and shLAMP-2A followed by selection with puromycin (Sigma) for 10 days.

\section{In vivo tumor allograft experiment and immunofluorescence on frozen tissue section}

LLC cells were injected subcutaneously into the flanks of 6-week-old C57BL/6 mice. Vehicle or ManA (5 $\mathrm{mg} / \mathrm{kg}$ ) was administered intraperitoneally every other day (Figure 1B, $\mathrm{n}=12$ per group). Vehicle, ManA (2 $\mathrm{mg} / \mathrm{kg}$ ), 17-AAG (25 mg/kg), anti-PD-1 mAb (5 and $15 \mathrm{mg} / \mathrm{kg}$ ), or their combination was administered intraperitoneally every other day (Figure $5 \mathrm{~A}, \mathrm{n}=6$ per group; Figure 6B, $\mathrm{n}=4$ per group). LLC cells expressing 
shLAMP-2A or carrying a control vector were injected subcutaneously into the flanks of 6-week-old C57BL/ 6 mice. Vehicle or ManA (5 mg/kg) was administered intraperitoneally every other day (Figure 5D, $\mathrm{n}=5$ per group). Tumor growth was measured with a caliper every other day using the formula, volume $=$ length $\mathrm{x}$ width ${ }^{2} \times 0.5\left(\mathrm{~mm}^{3}\right)$. Tumor tissues were removed at the experimental endpoint and fixed with $4 \%$ paraformaldehyde (Sigma). Tissues were dehydrated by serially incubating with $10 \%$ to $40 \%$ sucrose solution, and embedded in tissue freezing medium (Leica Microsystems, Wetzlar, Germany). Frozen blocks were cut into $50 \mu \mathrm{m}$ sections, blocked with $5 \%$ goat serum in PBST (0.03\% Triton X-100 in PBS), and then incubated for $3 \mathrm{~h}$ at room temperature (RT) with the following primary anti-Ki67 (Santa Cruz, California, Santa Cruz, CA), anti-CD31 (BD Biosciences, San Jose, CA), anti-HIF-1a (BD Transduction Laboratories, San Jose, CA), anti-GAPDH, anti-IKBa (Cell Signaling Technology, Danvers, MA), anti-LAMP2A, anti-PD-L1, anti-FOXP3, anti-aSMA (Abcam, Cambridge, UK), anti-CD4, anti-CD8a (BioLegend, San Diego, CA). Frozen blocks were washed with PBST and incubated with specific fluorescent secondary antibodies (Thermo Fisher Scientific) for $1 \mathrm{~h}$. Nuclei were staining with DAPI (Vector Laboratories, Burlingama, CA) and mounted with fluorescent mounting medium (Sigma). To detect the hypoxic area in the tumors, Hypoxyprobe- $1^{\mathrm{TM}}$ ( $60 \mathrm{mg} / \mathrm{kg}$, Natural Pharmacia International, Burlington, MA) was intravenously injected $90 \mathrm{~min}$ before tissue fixation. Tumors were harvested, sectioned and stained with MAb1antibody (Natural Pharmacia International, Burlington, MA) and a fluorescent secondary reagent.

\section{Western blot assay}

Proteins from cellular lysates were resolved on Tris-Glycine gel or NuPAGE ${ }^{\text {TM }} 4-12 \%$ Bis-Tris Protein Gels (Thermo Fisher Scientific) and transferred to nitrocellulose membranes (Whatman, Maidstone, England). Membranes were incubated overnight at $4{ }^{\circ} \mathrm{C}$ with primary antibodies specific for proteins as follows: anti-HIF-1a (BD Transduction Laboratories), anti-PCNA, anti-phospho-AKT (S473), anti-AKT, anti-ATG7, anti-LC3B, anti-TFEB, anti-cleaved caspase-3, anti-a-Tubulin, anti-EPS8 (Cell Signaling Technology), antiHer2, anti-ATP synthase $\beta$ (Thermo Fisher Scientific), anti-Hsp90a (Enzo Life Sciences, Farmingdale, NY), anti-LAMP1, anti- $\beta$-actin (Santa Cruz Biotechnology), anti-LAMP-2A, anti-LAMP-2B, and anti-Hsp90a (Abcam). Then, the membranes were incubated with horseradish peroxidase-conjugated mouse- or rabbitIgG (GenTex, Irvine, CA) for $1 \mathrm{~h}$ at RT and developed by using West Pico Chemiluminescent Substrate (Pierce, Woburn, MA). The proteins were detected by a digital image analyzer (ImageQuant LAS 4000, GE Healthcare, Buckinghamshire, UK), and the protein bands were obtained within linear range without saturation.

\section{Isolation of the lysosome-rich fraction}

Isolation of the lysosome-rich fraction from cells was performed using a kit from BioVision (K235-50) according to the manufacture's protocol. $2 \times 10^{7}$ cells were pelleted by centrifugation at $600 \mathrm{xg}$ for 10 min, added in $500 \mu \mathrm{l}$ of Lysosome Isolation Buffer, and incubated on ice for $2 \mathrm{~min}$. Cells were homogenized by passing 30 times through a Glass Dounce Homogenizer. The homogenate was transferred to a fresh tube with $500 \mu \mathrm{l}$ of Lysosome Enrichment Buffer, and the sample was centrifuged at $500 \mathrm{xg}$ for $10 \mathrm{~min}$ at $4{ }^{\circ} \mathrm{C}$. The supernatant was overlayed on the top of a prepared discontinuous 
density gradient and gradient centrifuged at $145,000 \times \mathrm{g}$ for $2 \mathrm{~h}$ at $4{ }^{\circ} \mathrm{C}$. The lysosome-rich fraction band was collected, mixed with two volumes of PBS, and centrifuged for $30 \mathrm{~min}$ at $18,000 \mathrm{xg}$. Isolated lysosomes were suspended in RIPA buffer, and protein concentration was determined.

\section{RNA isolation and RT-PCR}

Total RNA was extracted using a RNeasy Mini Kit (Qiagen, Valncia, CA) and reverse transcribed with a PrimeScript $^{\text {TM }}$ RT reagent Kit (Takara Bio Inc., Shiga, Japan). Real-time PCR was performed using an ABI PRISM 7300 Sequence Detection System using SYBR-Green PCR master mix (Applied Biosystems, Foster City, CA) with primer pairs targeting HIF-1a forward, ACCCTCTTCGTCGCTTCGG, and reverse, GCCTCTCCTCAGGTGGCTTG; GLUT1 forward, CAACACGGCCTTCACTGTCG, and reverse, ATGCCAGCGAGGCCTATGAG; PFK forward, CATTTATGTGGGTGCCAAAG, and reverse, ATGATGTTGGAGACGCTCAG; LDHA forward, CATCCTGGGCTATTGGACTCT, and reverse, TGTCCCAAAATGCAAGGAACA; HK2 forward, AAGGTAGAAATGGAGCGAGGT, and reverse, CCCGGAAATTTGTTCCTCCAA; LAMP1 forward, GTGTCTGCTGGACGAGAACA, and reverse, ATGAGGACGATGAGGACCAG; LAMP-2A forward, AACTTCCTTGTGCCCATAGC, and reverse, AGCATGATGGTGCTTGAGAC; LAMP-2B forward, AGAGTGTTCGCTGGATGATG, and reverse, TGCCAATTACGTAAGCAATCA; LAMP-2C forward, AAGGGTTCAGCCTTTCAATG, and reverse, ACAATTATAAGGAAGCCCAAGG; EPS8 forward, CAACCCAGAACAAGCCAGAT, and reverse TGCATTGGAAATCATCCTCA. IKBa forward, TCCTGAGCTCCGAGACTTTC, and reverse, CACGTGTGGCCATTGTAGTT; GAPDH forward, AATCCCATCACCATCTTCCA, and reverse TGGACTCCACGACGTACTC; GFAP forward, AGGAAGATTGAGTCGCTGGA, and reverse ACCTCCTCCTCGTGGATCTT; EEF1A1 forward, CTGTATTGGATTGCCACACG, and reverse GCAGCATCACCAGACTTCAA; RAC1 forward, TGGCTAAGGAGATTGGTGCT, and reverse TCGCTTCGTCAAACACTGTC; RICTOR forward CGGTTGTAGGTTGCCAGTTT, and reverse TCTTTCGGGTTTCATTCCAG; AKT1 TCTATGGCGCTGAGATTGTG, and reverse CTTAATGTGCCCGTCCTTGT; CTSA forward TTCTCTCCAGACCCACTGCT, and reverse GTCACGCATTCCAGGTCTTT; PHLPP1 forward ATCCTGGGCTACACCTTCCT, and reverse CCACAACCCCTTACTGCCTA; $\beta$-actin forward, GGATGTCCACGTCACACTTC, and reverse, CACTCTTCCAGCCTTCCTTC. Gene expression was normalized to that of the endogenous reference gene, $\beta$-actin.

\section{Lentivirus production}

HEK293T cells were transfected with pSIN-PAmCherry-KFERQ-NE expression vector (a gift from Shu Leong Ho, Addgene plasmid \#102365) and Virapower ${ }^{\mathrm{TM}}$ packaging mix (Thermo Fisher Scientific) using PolyFect Transfection reagent (QIAGEN, Hilden, Germany) according to the manufacturer's instructions. After $48 \mathrm{~h}$ incubation, supernatant containing lentivirus was harvested.

\section{CMA activity assay}


Cells transduced with a lentivirus carrying the PA-mCherry-KFERQ-NE were exposed under UV-A lamp (405 $\mathrm{nm}$ ) for $5 \mathrm{~min}$. After photoactivation of PA-mCherry-KFERQ-NE proteins, cells were treated with vehicle, ManA, cruentaren A, 6-AN, or 17-AAG under hypoxic conditions. Cells were fixed with $4 \%$ paraformaldehyde (Sigma) and nuclei were stained with DAPI (Vector Laboratories). CMA activity was assessed by counting the number of puncta in cells.

\section{Immunocytochemistry}

Cells were fixed with $4 \%$ paraformaldehyde (Sigma) and permeabilized with $0.1 \%$ Triton X-100. After blocking in $5 \%$ normal goat serum (Vector Laboratories, Burlingama, CA) for $1 \mathrm{~h}$, cells were incubated with primary anti-LAMP-2A, anti-HIF-1a (BD Transduction Laboratories), anti-ATP synthase $\beta$ (Thermo Fisher Scientific), anti-Hsp90a (Enzo Life Sciences), or anti-Flag (Sigma) overnight at $4{ }^{\circ} \mathrm{C}$. Cells were washed with PBS and incubated with specific fluorescent secondary antibodies (Thermo Fisher Scientific) for $1 \mathrm{~h}$. Nuclei were stained with DAPI (Vector Laboratories) and mounted with fluorescent mounting medium (Sigma). Colocalized area was quantified in four images using Image J.

\section{Transfection of siRNA and expression vectors}

Cells were transfected with scrambled siRNA, siLAMP-2A, siVPS4A, siVPS4B, siTFEB (Bioneer, Daejeon, South Korea), HIF-1 a P402A/P564A double mutant expression vector [28], Myr-p110a expression vector, TFEB-3 X Flag expression vector (kindly provided from Dr. Andrea Ballabio, Baylor College of Medicine), pcDNA3-FLAG-tagged wild-type hHsp90a expression vector (kindly provide from Dr. Leonard M. Neckers, National Cancer Institute), or a control vector using Lipofectamine RNAiMAX reagent (Thermo Fisher Scientific) and TransIT-LT1 Transfection reagent (Mirus Bio, Madison, WI) according to the manufacturer's instructions

\section{Immunoprecipitation}

HEK293T cells were lysed with protein lysis buffer (iNtRON, Daejeon, Korea) containing protease inhibitors (Sigma), and the lysates were incubated with anti-HIF-1a (BD Transduction Laboratories) or anti-ATP synthase $\beta$ (Thermo Fisher Scientific) at $4{ }^{\circ} \mathrm{C}$ for overnight. Slurry protein A/G agarose beads (Pierce, Woburn, MA) were added to reaction mixture, and the mixture was incubated for $3 \mathrm{~h}$ at $4{ }^{\circ} \mathrm{C}$. For confirmation of HIF-1a-Hsc70 interaction, cell samples were lysed in lysis buffer (50 mM Tris- $\mathrm{HCl}(\mathrm{pH} 7.5)$, $150 \mathrm{mM} \mathrm{NaCl}, 10 \mathrm{mM}$ sodium molybdate, $0.1 \% \mathrm{NP}-40$ ) containing protease inhibitors (Sigma). The lysates were incubated with anti-HIF-1a (BD Transduction Laboratories)-conjugated protein G plus agarose beads (Santa Cruz Biotechnology) for $3 \mathrm{~h}$ at $4{ }^{\circ} \mathrm{C}$. Following immunoprecipitation, the beads were washed four times with lysis buffer. Samples were denatured by adding sample buffer in beads and boiling for $10 \mathrm{~min}$. Co-immunoprecipitated proteins were detected by western blot analysis.

\section{Measurement of ATP}

The intracellular ATP concentration was measured using ATP Assay Kit (Abcam) according to the manufacturer's instructions. 


\section{Cell proliferation assay}

Cells were maintained in the absence or presence of ManA at indicated concentration in complete media under hypoxic conditions for 10 days. Media were changed every 2 days. Cells were fixed and stained with $0.5 \%$ crystal violet (Sigma). The quantitative image analyses were performed using ImageJ software.

\section{Measurement of oxygen consumption rate}

Oxygen consumption rate (OCR) was measured in a XF24 Extracellular Flux Analyzer (Seahorse Bioscience, North Billerica, MA, USA). Vehicle, ManA $(1 \mu \mathrm{M})$, oligomycin $(1 \mu \mathrm{M})$, CCCP $(5 \mu \mathrm{M})$, rotenone $(1$ $\mu \mathrm{M})$, or antimycin $\mathrm{A}(5 \mu \mathrm{M})$ was added at the indicated time points during OCR measurement. The OCR was normalized to the cell number and expressed as a ratio relative to baseline.

\section{Gene expression profiling}

MDA-MB-231 cells were treated with vehicle, ManA, geldanamycin, or 17-AAG under hypoxia or normoxia for $24 \mathrm{~h}$. Total RNA was extracted using a mirVana extraction kit (Thermo Fisher Scientific). RNA quality was assessed by electrophoresis. RNA samples were prepared and labelled for interrogation with Affymetrix GeneChip U133 plus 2.0 arrays (Thermo Fisher Scientific) to measure the expression of more than 54,000 probe sets and at least 47,000 transcripts and variants. The raw array data was normalized by RMA for further analysis.

\section{Luciferase refolding assay}

ManA and 17-AAG at indicated concentrations in DMSO were added to wells of a white, round-bottom 96well plate containing $50 \mu \mathrm{L}$ of cell culture media. Luciferase (pGL4.50-Luc; Promega, Madison, WI)expressing HEK293T cells were incubated for $10 \mathrm{~min}$ at $50^{\circ} \mathrm{C}$ in pre-warmed cell culture media until bioluminescence of luciferase was reduced to $1 \%$ of the initial counts. Cells were added to wells, and the plate was returned to the incubator for $1 \mathrm{~h}$. After $1 \mathrm{~h}, 100 \mu \mathrm{L}$ of luciferase substrate reagent (Promega; 75 $\mathrm{mM}$ tricine at $\mathrm{pH} 7.8,24 \mathrm{mM} \mathrm{MgSO}_{4}, 0.3 \mathrm{mM}$ EDTA, $2 \mathrm{mM}$ DTT, $0.313 \mathrm{mM}$ D-luciferin, 0.64 mM coenzyme A, $0.66 \mathrm{mM}$ ATP, $150 \mathrm{mM} \mathrm{KCl}, 10 \%$ Triton X-100, 20\% glycerol, and 3.5\% DMSO) was added to wells, and the bioluminescence was immediately measured ( $0.5 \mathrm{~s}$ integration time). Cells that were incubated in DMSO were used as $100 \%$ bioluminescence (i.e., DMSO $=100 \%$ refolding), and the relative refolding for each compound concentration was compared to that in DMSO.

\section{HS-10 resin binding assay}

Pig mammary proteins were extracted as previously reported[29]. Gamma-phosphate linked ATP Sepharose slurry was mixed with pig mammary lysate $(\mathrm{v} / \mathrm{v})$ for $1 \mathrm{~h}$ at RT. Following three washes with buffer A (50mM Tris-HCl pH 7.4, 1 mM DTT, $60 \mathrm{mM} \mathrm{MgCl}_{2}, 60 \mathrm{mM} \mathrm{KCl}, 1 \mu \mathrm{M}$ microcystin) plus $1 \mathrm{M} \mathrm{NaCl}$, the sepharose was mixed with increasing concentrations of HS-10, manassantin A, or ATP (100 mM), and the eluates were mixed with SDS sample buffer and heated for 5 minutes at $90^{\circ} \mathrm{C}$. Before binding to the 
HS-10 resin, each sample of lysate was pre-incubated with HS-10 or manassantin A for $1 \mathrm{~h}$ at RT. Following the pre-treatment, all samples were tumbled with $100 \mu \mathrm{L}$ of $50 \%$ slurry of resin. After $1 \mathrm{~h}$ of tumbling, the resin was washed 3 with a low stringency wash buffer $(50 \mathrm{mM}$ Tris- $\mathrm{HCl}, 60 \mathrm{mM} \mathrm{MgCl}, 60$ $\mathrm{mM} \mathrm{KCl}, 10 \mathrm{mM}$ citrate, $1 \mathrm{mM}$ DTT, pH 7.4). After the final wash, the buffer was completely aspirated, replaced with SDS sample buffer, and processed as above. Protein gel electrophoresis and silver staining were performed as previously reported[29].

\section{NMR spectroscopy}

Hsp90 constructs were prepared according to the previous report [30,31]. Backbone resonance assignments for the NTD (1-235) and MD (Middle domain, 293-554) proteins were obtained from the previously published data[30, 31]. For NMR titration experiments, $0.1 \mathrm{mM}^{15} \mathrm{~N}$-labeled Hsp90 NTD and Hsp90 MD were prepared. Both the Hsp90 NTD and Hsp90 MD were titrated with addition of two molar equivalents of mannassantin A in the presence of 10\% DMSO. The DMSO itself did not disrupt the 3D structure of the proteins since each resonance was well separated in the spectra as the spectrum in the buffer. We could not find any sign of protein aggregation in the 10\% DMSO solution. The chemical shift changes of ${ }^{1} \mathrm{H}$ and ${ }^{15} \mathrm{~N}$ resonance were measured in a series of $2 \mathrm{D}\left[{ }^{1} \mathrm{H},{ }^{15} \mathrm{~N}\right]$ - TROSY spectra, which were recorded at $298 \mathrm{~K}$ using a Bruker AVANCE III 600 spectrometer equipped with a cryogenic probe. All NMR spectra were processed using the program NMRPipe[32] and were analyzed with the program NM View[33].

\section{SPR assay}

The GST-CTD (C-terminal domain, 464-732) protein of Hsp90 was prepared. Briefly describing, proteins were expressed in LB medium in the $E$. coli host BL21 with induction at $37^{\circ} \mathrm{C}$ for $4-6 \mathrm{~h}$. The GST fused Cterminal domain was purified using the GST-affinity column, GSTPrep FF16/60 (GE Healthcare, Chicago, IL) followed by anion exchange chromatography, HiTrap Q HP and size exclusion chromatography, Sephacryl S100HR column. SPR assay was performed in the Biacore X100 instrument (GE Healthcare). The Anti-GST antibody ( $40 \mu \mathrm{l}, 0.6 \mathrm{mg} / \mathrm{ml}$ ) was immobilized on the Biacore sensor chip CM5 (GE Healthcare) using GST-capture kit (GE Healthcare). Activation of the CM5 chip was achieved EDC/NHS solution using a flow rate of $5 \sim 10 \mu \mathrm{l} / \mathrm{min}$. The immobilization of the Anti-GST antibody gave the response units $(\mathrm{RU})$ of 6,000-7,000. The minor fraction of high affinity sites of the polyclonal anti-GST antibody was blocked with recombinant GST $(95 \mu \mathrm{l}, 20 \mu \mathrm{g} / \mathrm{ml})$ at a flow rate of $20 \mu \mathrm{l} / \mathrm{min}$ before capturing of the GST-CTD protein on the antibody. The reference surface should be prepared in the same way as the active surface. The capturing of GST-CTD $(55 \mu \mathrm{l}, 100 \mu \mathrm{M})$ on the active cell was achieved by the manufacturer's guide using the capturing protocol that is implemented in the instrument. The surface regeneration of the immobilized chip was achieved with $40 \mu \mathrm{l}$ of $10 \mathrm{mM}$ glycine- $\mathrm{HCl}(\mathrm{pH} 2.2)$ injection at a flow rate of $20 \mu \mathrm{l} / \mathrm{min}$ across all flow cells. A serial dilution of manassantin $A$ at $0,5,10,20,50$, and $100.0 \mu \mathrm{M}$ in $1 \times$ HBS-EP+ buffer (GE Healthcare) supplemented with $2 \%$ DMSO were injected over the GST-CTD captured and reference surfaces at contact time, $180 \mathrm{~s}$ and flow rate, $10 \mu \mathrm{l} / \mathrm{min}, 25^{\circ} \mathrm{C}$. To correct the solvent effect originated from DMSO, the step of solvent correction was included during binding 
experiments. Eight DMSO solutions ranged from $1.5 \%$ to $2.8 \%$ were prepared and were run at the stage of solvent correction cycle in the assay procedure. The equilibrium dissociation constant $\left(\mathrm{K}_{\mathrm{d}}\right)$ of manassantin A to the Hsp90 C-terminal domain were analyzed by Evaluation software implemented in the Biacore X100 instrument using the fit models: 1:1 Binding kinetic and Steady State Affinity.

\section{Flow cytometry}

To facilitate analysis of lymphoid compartments, tumors were digested with $50 \mathrm{mg} / \mathrm{ml}$ collagenase $D$ (Roche) and $10 \mathrm{mg} / \mathrm{ml}$ DNase (Roche) per tumor in RPMI 1640 for $1.5 \mathrm{~h}$ at $37^{\circ} \mathrm{C}$. After pelleting the cells, they were incubated for 5 minutes with ACK lysing buffer (Lonza, Walkersville, MD) and then washed and resuspended in Hank's balance salt solution (HBSS) (Lonza, Walkersville, MD). Single-cell suspensions of tumors were filtered through a $40 \mu \mathrm{M}$ nylon strainer (Falcon) and blocked for $10 \mathrm{~min}$ on ice with CD16/CD32 Fc block (clone 93) (eBioscience). To detect tumor infiltrated T cell subtypes whole single-cell suspensions were stained with the cell surface markers, PE-cyanine7 CD3e (Thermo Fisher Scientific), PerCP CD8a, (BD Transduction Laboratories), and FITC-CD4 (Invitrogen) antibodies in a FACS buffer, and then analyzed by flow cytometer (FACS Canto Il; BD Biosciences). Tumor samples were stained with 1 $\mathrm{mg} / \mathrm{ml}$ DAPI to exclude dead cells and the flow cytometric analyses were performed using a BD LSR II flow cytometer with FACSDiva ${ }^{\mathrm{TM}}$ software.

\section{TUNEL assay}

Tumor sections were stained using DeadEnd ${ }^{\mathrm{TM}}$ Fluormetric TUNEL System (Promega) according to the manufacturer's instructions.

\section{Patient survival data}

The correlation between gene expression and patient outcome has been identified in each cancer type using Prediction of Clinical Outcomes from Genomic Profiles (Precog)[34]. To determine the correlation between patient overall survival and gene expression, gene expression datasets of GE011969 (only datasets with adenocarcinoma and squamous cell carcinoma patients were included) and TCGA PanCancer Atlas were downloaded from the Gene Expression Omnibus (GEO) repository and cbioportal for lung adenocarcinoma and invasive breast carcinoma. Gene expression of HIF1A, TFEB, and HSP90AA1, or combined expression of TFEB/HIF1A and HSP90AA1/HIF1A was compared with overall patient survival. To acquire statistical significance, gene expression was divided based on median expression value, while quartile cut points were used for single gene expression of TCGA datasets (smallest $25 \%$ for TFEB as a negative prognostic factor, top $25 \%$ of HSP90AA1 as a positive prognostic factor). Kaplan-Meier curves were plotted using GrapPad Prism 8.4.2 and $p$ values were determined by Log-rank test.

\section{Pharmacokinetics of ManA}


C57BL/ 6 mice were administered ManA at doses of $2 \mathrm{mg} / \mathrm{kg}$ (iv; intravenous injection) or $5 \mathrm{mg} / \mathrm{kg}$ (po; per oral administration) and venous blood samples were collected at $0.5,1,2,4,8$, and $24 \mathrm{~h}$ post-dose. A $20 \mu \mathrm{L}$ of plasma was separated by centrifugation and stored at $-80^{\circ} \mathrm{C}$ until analysis. A $20 \mu \mathrm{L}$ aliquot of plasma samples was deproteinized by addition of $100 \mu \mathrm{L}$ cold methanol (containing berberine $0.1 \mathrm{ng} / \mathrm{mL}$ as internal standard) and centrifuged at 13,200 rpm for $10 \mathrm{~min}$. A $10 \mu \mathrm{L}$ aliquot of the supernatant was injected into Agilent 6470 LC-MS/MS system. Separation was performed on a Synergy Polar RP column ( $4 \mu \mathrm{m}$ particle size, $2.0 \mathrm{~mm} \times 150 \mathrm{~mm}$; Phenomenex, Torrance, CA, USA) using a mobile phase that consisted of methanol and water $(85: 15, \mathrm{v} / \mathrm{v})$ with $0.1 \%$ formic acid at a flow rate of $0.2 \mathrm{ml} / \mathrm{min}$. Quantification was carried out using multiple reaction monitoring (MRM) at $\mathrm{m} / \mathrm{z} 750.4 \rightarrow 259.2$ for ManA and $\mathrm{m} / \mathrm{z} 336.0 \rightarrow 320.0$ for berberin (internal standard) in positive ionization mode and collision energy of $25 \mathrm{eV}$.

The pharmacokinetic parameters were determined by the non-compartmental analysis (WinNonlin ${ }^{\circledR}$ 5.1; Pharsight, Mountain View, CA, USA). The maximum plasma concentration (Cmax) and time to reach $\mathrm{C}_{\max }$ $\left(T_{\max }\right)$ values were obtained from the plasma concentration-time curves. The area under the plasma concentration-time curve from zero to infinity (AUC) was calculated by the trapezoidal-extrapolation method. Oral bioavailability (BA) was calculated as follows: BA $(\%)=$ AUCp.o. normalized by po dose/AUCi.v. normalized by iv dose $\times 100$.

\section{Plasma and metabolic stability of Man A}

ManA stock solution (10 mM dissolved in methanol) was diluted with potassium phosphate buffer (pH7.4) to make a concentration of $1 \mu \mathrm{M}$. A $10 \mu \mathrm{L}$ aliquot of this solution was then added to $80 \mu \mathrm{L}$ aliquot of $100 \mathrm{mM}$ potassium phosphate buffer $(\mathrm{pH7.4)}$ containing human or mouse liver microsomes ( $80 \mu \mathrm{L}$ of $0.625 \mathrm{mg}$ protein $/ \mathrm{ml}$ phosphate buffer) and incubated at $37^{\circ} \mathrm{C}$ for $10 \mathrm{~min}$ before adding of 10 $\mu \mathrm{L}$ of an NADPH regenerating system to start the reaction. Reactions were stopped at $0,15,30,45$, and 60 min time-points by the addition of $200 \mu \mathrm{L}$ cold methanol (containing $0.1 \mathrm{ng} / \mathrm{mL}$ berberine as internal standard) and vortexed for $15 \mathrm{~min}$ and centrifuged at $16,000 \times \mathrm{g}$ for $5 \mathrm{~min}$. A $5 \mu \mathrm{L}$ aliquot of the supernatant was injected into the LC-MS/MS system. Positive control study using $1 \mu \mathrm{M}$ metformin (for high microsomal stability) and $1 \mu \mathrm{M}$ propranolol (for low microsomal stability) was also performed to ensure the system feasibility. For plasma stability, $100 \mu \mathrm{L}$ of mouse or human plasma containing $1 \mu \mathrm{M}$ of each compound (Man A, metformin, or propranolol) was incubated for $2 \mathrm{~h}$ at $37^{\circ} \mathrm{C}$ on a shaking incubator at $300 \mathrm{rpm}$. Reactions were stopped at 0,30,60, 90, and $120 \mathrm{~min}$ time-points by the addition of $200 \mu \mathrm{L}$ cold methanol (containing $0.1 \mathrm{ng} / \mathrm{mL}$ berberine as internal standard). Thereafter, the mixture was vortexed for $15 \mathrm{~min}$ and centrifuged at $16,000 \times \mathrm{g}$ for $5 \mathrm{~min}$. After centrifugation, a $5 \mu \mathrm{L}$ aliquot was injected into the LC-MS/MS system. The ratio of peak area of test compound /internal standard was used to determine the \% remaining of each compound over time. The half- life and \% compound remaining after $60 \mathrm{~min}$ were then calculated.

\section{Caco-2 permeability}


Caco-2 cells (passage no 42-43; purchased from ATCC, Rockville, MD, USA) were seeded on collagencoated 12-transwell membranes at a density of $5 \times 10^{5}$ cells $/ \mathrm{mL}$ and maintained at $37^{\circ} \mathrm{C}$ in a humidified atmosphere with $5 \% \mathrm{CO} 2 / 95 \%$ air for $21 \mathrm{~d}$. The culture medium was replaced every $2 \mathrm{~d}$ and the permeability assay was conducted as previously described. Briefly, HBSS medium containing marker compounds (caffeine, propranolol, ofloxacin, atenolol, and paclitaxel) $(2,5,10,50 \mu \mathrm{M}$, and $20 \mu \mathrm{M}$, respectively) or ManA $(20 \mu \mathrm{M})$ was added to the donor side and aliquots $(100 \mu \mathrm{L})$ from the receiver side were collected at $15,30,45$, and $60 \mathrm{~min}$ and stored at $-80^{\circ} \mathrm{C}$ until further analysis. The thawed samples were added to $300 \mu \mathrm{L}$ methanol (containing berberine as internal standard) and centrifuged at 13,200 rpm for $10 \mathrm{~min}$. A $5 \mu \mathrm{l}$ aliquot of the supernatant was injected into the LC-MS/MS system.

For the permeability calculation, the transport rate of ginsenosides and marker compounds was calculated from the slope of the regression line from the mean permeated amounts vs. incubation time plot. The apparent permeability (Papp) was calculated from the following equation:

$\operatorname{Papp}\left(10^{-6} \mathrm{~cm} / \mathrm{s}\right)=\frac{\text { transport rate }(\mathrm{nmol} / \mathrm{min})}{\text { concentration }(\mu M) \times \text { area }\left(\mathrm{cm}^{2}\right) \times 60 \mathrm{~s}}$.

\section{Statistical analysis}

All data were expressed as mean \pm standard deviations (SD) from at least 3 samples. Statistical comparisons were analyzed using the Student's $t$-test. Differences with $p<0.05$ and $p<0.01$ were considered statistically significant and highly significant, respectively.

\section{Results}

\section{Manassantin A inhibits tumor growth and HIF-1a protein expression not through pVHL-mediated proteasomal pathway}

To understand the mechanism by which ManA (Figure 1A) exerts its anti-tumor activity, we first tested the effect of ManA on tumor growth using tumor allograft experiment. Lewis lung carcinoma (LLC) cells were inoculated in the mouse flank subcutaneously (s.c.), and the mice were treated with intraperitoneal (i.p.) injection of ManA ( $5 \mathrm{mg} / \mathrm{kg}$ ) every other day. ManA significantly inhibited tumor growth (Figure 1B) and decreased tumor weight (Figure 1C). In addition, the body weight was not significantly changed (Figure S1A), indicating there was no gross toxicity by ManA injection on mice. ManA significantly decreased microvessel density (MVD), hypoxic regions, and HIF-1a expression (Figure 1D).

Proteasomal degradation of HIF-1a is mediated by PHDs and pVHL E3 ubiquitin ligase under normoxia [12]. Since we demonstrated ManA is a novel inhibitor of HIF-1a under hypoxia [24], we first examined the effect of ManA on HIF-1a expression. ManA decreased the HIF-1 a protein expression time- and dosedependently (Figure 1E and S1B) without altering the mRNA expression of HIF-1a (Figure S1C). In addition, ManA showed the same effect on HIF-1 a protein expression induced by desferrioxamine (DFO), an iron (III) chelator (Figure S1D). 
We next examined whether ManA regulates HIF-1a stability through the ubiquitination-proteasomal degradation pathway under hypoxic conditions. ManA increased HIF-1a degradation in the presence of MG132, a proteasome inhibitor, under hypoxic conditions (Figure 1F). Since HIF-1a is recognized and hydroxylated by PHDs at both Pro402 and Pro564 under normoxic conditions, subsequently ubiquitinated, and degraded by proteasome [35], we examined HIF-1a protein expression by double mutation of P402A/P564A or treatment of dimethyloxalylglycine (DMOG), a proline hydroxylation (PHD) inhibitor, in the presence of ManA. ManA decreased HIF-1 a protein expression in both P402A/P564A double mutation and DMOG treatment (Figure 1G). In addition, ManA significantly enhanced the HIF-1a degradation rate induced by reoxygenation (Figure $1 \mathrm{H}$ ). These results indicate that ManA degrades HIF1a protein independent of the standard PHDs-pVHL-mediated proteasomal degradation under hypoxic conditions.

\section{Manassantin A inhibits tumor growth and HIF-1a protein expression through lysosomal pathway of autophagy}

Because HIF-1a proteins are degraded by CMA under hypoxic conditions [8], we investigated whether ManA upregulates the degradation of CMA substrates in vivo. Interestingly, the treatment of ManA significantly decreased cell proliferation ( $\mathrm{Ki} 67^{+}$cells) and CMA substrates (GAPDH and IKBa) expression in the tumor tissues from LLC mouse allograft experiment of Figure 1B, whereas LAMP-2A expression was increased in lysosomes, as evidenced by co-localization with lysosomal protein LAMP1 (Figure 2A). These results suggested that ManA might be related to CMA activation. To examine whether ManA is related to CMA activation, the CMA activity in HEK293T cells after ManA treatment was measured by using PAmCherry-KFERQ-containing fluorescent reporter. The reporter protein shows the fluorescence pattern from diffuse to puncta when CMA is activated [36]. ManA and 6-aminonicotinamide (6-AN), a known CMA activator [8], increased CMA activity under hypoxic conditions (Figure 2B). To investigate the possibility that the inhibition of macroautophagy causes the compensatory upregulation of CMA, we examined the changes in CMA activity using 3-methyladenine (3-MA), a selective inhibitor of macroautophagy [37]. 3-MA did not alter CMA activity under hypoxic conditions (Figure 2B). Furthermore, we validated the effect of CMA on ManA-mediated HIF-1 a degradation using autophagy inhibitors (Figure $2 \mathrm{C}$ and 2D). HIF-1 a degradation induced by ManA was blocked by chloroquine (Figure 2C) and bafilomycin (Figure 2D)-under hypoxic conditions. In addition, ManA-induced inhibition of GLUT1 mRNA expression was rescued by chloroquine (Figure 2E) or bafilomycin (Figure 2F). Taken together, these results suggest that ManA inhibits tumor growth through a mechanism involving CMA activation.

\section{Activation of chaperone-mediated autophagy by manassantin A induces HIF-1a degradation and inhibits cancer cell proliferation under hypoxic conditions.}

Since chloroquine and bafilomycin can inhibit both CMA and macroautophagy [38], we investigated whether macroautophagy was involved in HIF-1 a protein degradation induced by ManA. We examined HIF-1a protein expression by treatment with 3-MA, a selective inhibitor of macroautophagy, in the presence of ManA. 3-MA decreased autophagic LC3-II flux but had no effect on HIF-1a protein 
degradation induced by ManA (Figure 3A). Since the knockout of ATG7 impairs the formation of autophagosomes in macroautophagy [39], we employed ATG7 knockout (KO) systems. ManA did not alter HIF-1a mRNA expression (Fig. S2A) but degraded HIF-1 a proteins in ATG7 KO cells under hypoxic conditions (Figure 3B). Moreover, the knockdown of VPS4A and VPS4B, which is particularly suitable for distinguishing between CMA and microautophagic degradation [2], did not reverse ManA-induced HIF-1a degradation (Figure S2B). We also confirmed the effects of ManA on the mRNA and protein levels of GAPDH, IKBa, and EPS8, which are known as CMA substrates [40]. The mRNA levels were not significantly altered, but the protein levels were reduced (Figures $3 \mathrm{C}$ and S2C). Moreover, treatment with chloroquine increased HIF-1a, GAPDH, and EPS8 levels in lysosomes isolated from ManA-treated cells (Figure 3D). Next, CMA activators AR7, 6-AN, and paraquat inhibited HIF-1a protein expression in a similar manner to ManA under hypoxic conditions (Figures S2D-S2F).

To further investigate whether CMA network components are responsible for HIF-1a degradation by ManA, we examined genes involved in CMA by qRT-PCR. ManA increased only the LAMP-2A mRNA level (Figure S2G and S2H). In addition, western blot analysis showed that ManA increased LAMP-2A expression with HIF-1a protein degradation under hypoxic conditions, while ManA had lesser effects on the degradation under LAMP-2A silencing (Figure 3E). Although LAMP-2A is a rate-limiting step of the CMA pathway [2], we further measured the mRNA and protein levels of other LAMP such as LAMP1, LAMP-2B, and LAMP-2C. The mRNA and protein levels of all the other LAMP except for LAMP-2A were not altered by ManA (Figures S2G and S2I). As LAMP-2A is particularly more relevant than other LAMP proteins for distinguishing CMA from other autophagic degradation pathways [41], thus, the degradation of HIF-1 a proteins by ManA is mediated through LAMP-2A. We next examined the interactions of HIF-1a with LAMP-2A under hypoxic conditions using immunoprecipitation (IP) assay and IF staining. ManA facilitated the interaction of HIF-1a with LAMP-2A (Figure 3F, 3G, and S2J). These results showed that ManA degrades HIF-1a protein through LAMP-2A-dependent activation of CMA.

To demonstrate the metabolic response of HIF-1a inhibition by CMA activation in vitro, we examined HIF1a-target glycolytic enzyme genes (GLUT1, HK2, PFK, and LDHA) by ManA treatment under hypoxic conditions using qRT-PCR. The mRNA expression of target genes was significantly decreased by ManA treatment under hypoxic conditions (Figure $3 \mathrm{H}$ ). To exclude the possibility that glycolytic inhibition by ManA promotes CMA activity, we confirmed CMA activity using the glycolytic inhibitor 2-deoxy-D-gucose (2-DG) under hypoxic conditions. 2-DG did not increase CMA activity (Figure S2K). These findings indicate that CMA activation is not a consequence of glycolytic inhibition in response to ManA.

We then investigated intracellular ATP and cell proliferation as the functional outcome of HIF-1a inhibition in vitro. We found that the intracellular ATP levels were significantly decreased by ManA treatment under hypoxic conditions (Figure S2L). In addition, ManA inhibited cancer cell proliferation and increased the cleaved caspase-3 expression in HEK293T, MDA-MB-231, A549, and LLC cells under hypoxic conditions (Figure $3 \mathrm{I}$ and S2M). However, siRNA-mediated LAMP-2A knockdown prevented ManA-induced cancer cell growth inhibition and apoptosis under hypoxia (Figures $3 \mathrm{~J}$ and $3 \mathrm{~K}$ ). These 
results suggest that CMA-mediated HIF-1a degradation by ManA induces metabolic stress by inhibiting glycolysis and cell proliferation under hypoxic conditions.

\section{Manassantin A inhibits Hsp90 activity}

To understand the molecular mechanism by which ManA activates CMA for HIF-1a degradation to lysosome, we analyzed the global gene expression pattern in cells treated with ManA. We first used Significant Analysis of Microarrays (SAM) [42] to identify unique probe sets of which expression was consistently changed by ManA. Then, we analyzed the upregulated and downregulated genes for interrogating the Connectivity Map (CMAP) [43] to identify associated compounds. The CMAP analysis showed that ManA induced a highly similar gene expression pattern to several heat shock protein 90 (Hsp90) inhibitors, including geldanamycin and 17-(allylamino)-17-demethoxygeldanamycin (17-AAG) (Figure 4A, Table S1). This result suggests that there is correlation between ManA with Hsp90, which is involved in the lysosomal degradation of HIF-1a by inhibiting the Hsp90 function.

Since heat-denatured firefly luciferase requires functional Hsp90 to refold and produce bioluminescence $[44,45]$, we examined the refolding efficiency by ManA using intracellular luciferase refolding assay [45]. ManA significantly inhibited the refolding of luciferase up to $10 \mu \mathrm{M}$ in a dose dependent manner similar to 17-AAG (Figure 4B), which suggests that ManA plays a role as an Hsp90 inhibitor.

To determine whether ManA inhibits Hsp90 by direct interaction, we performed series of biochemical and biophysical experiments. When ATP-resin bound Hsp90 was treated with HS-10 (a Hsp90 N-terminal ATPbinding site inhibitor) [29], HS-10 eluted Hsp90 from the ATP-resin, whereas ManA did not elute Hsp90 from the ATP-resin (Figure S3A, left). We next used HS-10 immobilized on resin (HS-10 resin) that binds to Hsp90 N-terminus in pull-down assays. When the untethered HS-10 was pre-incubated in lysates, the free HS-10 prevented the HS-10 resin from pulling down Hsp90 N-terminus by competition (Figure S3A, right). We found that ManA did not eliminate binding of Hsp90 to the HS-10 resin when the lysate was pretreated with ManA, suggesting that ManA did not directly interact with Hsp90-N-terminus.

To further confirm that there is no direct ManA-Hsp90 interaction involving other Hsp90 domains, we performed Transverse Relaxation-Optimized Spectroscopy (TROSY) Nuclear Magnetic Resonance (NMR) experiments with each of the N-terminal and middle domain of Hsp90 (Figure S3B) and Surface Plasma Resonance (SPR) experiments using a GST-fused C-terminal domain of Hsp90 (Figure S3C). These biophysical experiments showed that there was no direct interaction of ManA to any domains of Hsp90. These results indicate that ManA modulates Hsp90 function in an indirect manner, which is distinct from other conventional Hsp90 inhibitors.

\section{Disrupting the $\mathrm{Hsp} 90 / \mathrm{F}_{1} \mathrm{~F}_{0}-\mathrm{ATP}$ synthase interaction by manassantin A promotes CMA by inhibiting the Hsp90 function and increasing TFEB nuclear localization.}

Various co-chaperones associate dynamically with Hsp90 during the chaperone cycle for client protein maturation. $F_{1} F_{0}$-ATP synthase has been implicated in $\mathrm{Hsp} 90$ chaperone function as a co-chaperone of 
Hsp90-client protein complexes. Disruption of the Hsp90/ $\mathrm{F}_{1} \mathrm{~F}_{0}$-ATP synthase complex results in the degradation of a subset of Hsp90 clients [46, 47]. To determine whether ManA inhibits Hsp90 function by disrupting the interaction of $\mathrm{Hsp} 90$ and $\mathrm{F}_{1} \mathrm{~F}_{0}$-ATP synthase, we performed immunoprecipitation assay with $F_{1} F_{0}$-ATP synthase and Hsp90. Hypoxia increased the formation of Hsp90/ $F_{1} F_{0}$-ATP synthase complex (Figure $4 \mathrm{C}$ ), supporting the role of $\mathrm{F}_{1} \mathrm{~F}_{0}$-ATP synthase as a co-chaperone for Hsp90. Interestingly, ManA effectively disrupted their interaction in a dose-dependent manner, whereas MA02 (an inactive ManA analog) [24] did not affect the Hsp90/ $\mathrm{F}_{1} \mathrm{~F}_{0}$-ATP synthase interaction under hypoxic conditions (Figure $4 C$ ). To confirm the effects on the interaction of $H s p 90$ and $F_{1} F_{0}-A T P$ synthase by ManA, we performed IF staining with their antibodies. Expression of $\mathrm{F}_{1} \mathrm{~F}_{0}$-ATP synthase was localized near the nucleus, while the expression level did not change upon hypoxic exposure or ManA-treatment (Figure 4C and 4D). Hsp90 was co-localized with $F_{1} F_{0}$-ATP synthase in the surroundings of the nucleus in both normoxic and hypoxic conditions, but ManA decreased the co-localization of $\mathrm{F}_{1} \mathrm{~F}_{0}$-ATP synthase with Hsp90 (Figure 4D, yellow color in merged images). Since disruption of the Hsp90/ $F_{1} F_{0}$-ATP synthase interaction induces mitochondrial dysfunction via inhibition of $F_{1} F_{0}$-ATP synthase $[46,48]$, we measured oxygen consumption rate to evaluate the mitochondrial dysfunction caused by ManA. As expected, ManA decreased mitochondrial oxygen consumption rates (Fig.4E). These results suggest that ManA inhibits Hsp90/ $F_{1} F_{0}$-ATP synthase (chaperon/co-chaperon) interaction.

Next, we examined whether disruption of $\mathrm{Hsp} 90 / \mathrm{F}_{1} \mathrm{~F}_{0}$-ATP synthase complex is related to CMA activity and HIF-1 1 degradation. Cruentaren $A$ is a known selective inhibitor of $F_{1} F_{0}$-ATP synthase [47]. The effects of cruentaren A were similar to those of ManA, including CMA activation and degradation of HIF1 a proteins (Figure S3D and S3E). Considering that the disruption of Hsp90/ $\mathrm{F}_{1} \mathrm{~F}_{0}-\mathrm{ATP}$ synthase interaction by cruentaren A inhibits Hsp90 activity, ManA could regulate Hsp90 client proteins via inhibition of Hsp90 function. We found that expression of a Hsp90 client protein Her2/Neu was decreased by ManA (Figure S3F), and phospho-AKT regulated by Hsp90 was also decreased upon ManA treatment (Figure 4F). It has been reported that AKT phosphorylation represses TFEB activation by inhibition of TFEB nuclear localization [49]. To confirm whether ManA-mediated AKT inhibition modulates TFEB nuclear localization, we investigated the subcellular localization of TFEB using IF staining. TFEB nuclear localization was increased by ManA and MK-2206, an AKT inhibitor, under hypoxic conditions (Figure $4 \mathrm{G}$ and S3G). In addition, activation of AKT by overexpression with myristolated-PI3Kp110a decreased TFEB nuclear translocation even in the presence of ManA (Figure 4G and S3G). To further confirm the role of TFEB in ManA-mediated HIF-1a protein degradation, we employed TFEB siRNA. TFEB silencing inhibited HIF-1a protein degradation by ManA under hypoxic condition (Figure $4 \mathrm{H}$ ). We further confirmed whether ManA-mediated AKT inhibition is associated with a decrease in glycolytic enzyme levels. The overexpression of myristolated-PI3Kp110a mitigated the ManA-induced suppression of glycolytic enzyme genes, specifically, increasing the mRNA expressions of GLUT1, HK2, and LDHA to levels similar to those of the control group (Figure 4I). Thus, these data collectively demonstrate that ManA-mediated AKT inhibition is required for TFEB nuclear localization and the suppression of glycolytic enzyme expression. 
Next, we examined whether Hsp90 inhibition activates CMA process using PAmCherry-KFERQ-containing fluorescent reporter. In agreement with a previous report on increased CMA activity by Hsp90 inhibitors [50-52], ManA as well as 17-AAG-treated cells increased CMA activity under hypoxic conditions (Figure 4J). We also observed that the overexpression of Hsp90 reversed the inhibitive effects of ManA on HIF-1a protein levels in HEK293T cells (Figure 4K), as well as in MCF-7 and Huh7 cancer cells, in which CMA is involved in cancer cell growth [53] (Figures S3H) and ManA-induced CMA activation was decreased by overexpression of Hsp90 in MCF-7 and Huh7 cells (Figure S3I), suggesting CMA is regulated by Hsp90 under hypoxia.

We also sought to determine whether ManA increases the binding of chaperone Hsc70 to HIF-1a to form a chaperone complex. Considering that CMA substrates interact with chaperone Hsc70 for their delivery to lysosomes [54], ManA may promote the binding of HIF-1a into a chaperone complex for its subsequent lysosomal degradation. We confirmed that treatment with ManA increased the binding of HIF-1a to Hsc70 (Figure 4L). These results suggest that the ManA-mediated inhibition of Hsp90 enhances the CMA pathway by disrupting the interaction between $\mathrm{Hsp} 90$ and $\mathrm{F}_{1} \mathrm{~F}_{0}$-ATP synthase and promotes the interaction of HIF-1 a with Hsc70, leading to a degradation of HIF-1a in lysosomes under hypoxic conditions.

\section{Manassantin A reduces tumor growth via Hsp90 inhibition, but LAMP-2A suppression prevents ManA- induced tumor regression}

To confirm whether the modulation of ManA-induced Hsp90 inhibition and LAMP-2A activation affect tumor growth in vivo, we tested the therapeutic effects of ManA in combination with 17-AAG, a specific Hsp90 inhibitor. Tumor masses treated with either 17-AAG or ManA were significantly smaller than those of control. However, their combination did not synergistically enhance the anti-tumor effects of ManA in mice (Figure $5 \mathrm{~A}$ ). Body weights were not markedly changed in any of the treatment groups, indicating no gross toxicity by ManA and/or 17-AAG (Figure 5B). In addition, we confirmed the expression of CMA substrates in ManA, 17-AAG, or vehicle-treated LLC allograft tumors. The expression of CMA substrates (HIF-1a, GAPDH, and IKBa) was significantly decreased in tumor tissues by treatment with the aforementioned drugs (Figure $5 \mathrm{C}$ ). Collectively, these data suggest that ManA decreases tumor growth through Hsp90 inactivation. We further investigated whether LAMP-2A mediates ManA-induced tumor suppression using an LLC allograft mouse tumor model. The tumors in mice injected with LAMP-2Aknockdown LLC cells were not significantly smaller (Figure 5D) and did not exhibit lower levels of CMA substrates in response to ManA compared with those in the control group (Figure 5F). These results indicate the CMA-dependent role of ManA in tumor growth.

\section{Combinational therapy of manassantin A and anti-PD-1 monoclonal antibody augments anti-tumor growth effects.}

Interestingly, we found that ManA decreased PD-L1 expression in the tumors from LLC mouse allograft experiment of Figure 1B (Figure 6A). Increased PD-L1 expression in various cancer cells has been 
associated with increased HIF-1 levels and the downregulation of T-cell function [55, 56]. Furthermore, PDL1 is a direct target of HIF-1a in cancer cells and microenvironment cells [55]. Accordingly, we sought to determine whether PD-L1 is regulated through the ManA-mediated Hsp90/HIF-1a pathways. The knockdown of HIF-1a attenuated the ManA-induced upregulation of PD-L1 in HEK293T and LLC cells (Figure S4A), whereas the overexpression of Hsp90 reversed the ManA-induced downregulation of PD-L1 and HIF-1a in these cells (Figure S4B). Therefore, the result suggests that CMA activator could inhibit PDL1 expression through HIF-1a degradation.

To investigate whether CMA activators enhance the response to anti-PD-1 antibody on tumor growth, we tested therapeutic effects of ManA in combination with anti-PD-1 antibody. When LLC tumor-bearing mice were treated with ManA (2 mg/kg), anti-PD-1 mAb (5 and $15 \mathrm{mg} / \mathrm{kg}$ ), or their combination, the tumor volume (Figure 6B) was reduced in the combination therapy group as compared with the monotherapy or the control group. No marked changes in body weight were observed in any of the treatment groups, indicating there was no gross toxicity (Figure S4C).

Tumor microenvironment consists of various stromal, immune, and inflammatory cells. Especially, subpopulation of lymphocytes plays major role in either promoting or regressing tumor growth [57]. To investigate whether the ManA and anti-PD-1 mAb combination infuences tumor infiltrating lymphocytes (TILs), we determined the levels of cytotoxic and regulatory lymphocytes in tumor tissues. We observed that the number of cytotoxic $\mathrm{CD} 8$-positive $\left(\mathrm{CD} 8^{+}\right) \mathrm{T}$ cells was significantly increased in combination therapy (Figure 6C). Next, we measured the level of regulatory T cells (Tregs) as it enables tumor growth by suppressing the proliferation of effector T-lymphocytes. We co-stained tumor tissues with anti-CD4 and anti-FOXP3 antibodies to detect Tregs. The IF images showed that the FOXP3 level was significantly reduced in combination therapy (Figure $6 \mathrm{C}$ ). We did not find any significant changes in the level of CD $4^{+}$ lymphocytes (Figure 6C). These results indicate that anti-tumor activity in combination therapy is enhanced by infiltration of $\mathrm{CD}^{+}$cells and decrease of Tregs in tumor. Moreover, the immunofluorescence imaging and FACS analysis results showed that ManA decreased PD-L1 levels and increased the number of $\mathrm{CD}^{+} \mathrm{T}$ cells in the tumor tissues (Figures S4D and S4E), suggesting that the ManA-mediated downregulation of PD-L1 boosts T-cell mediated cytotoxicity.

To determine the effects of CMA activaton induced by ManA on cell proliferation and angiogenesis in vivo, we performed immunohistochemistry on the tumors from untreated and treated groups.

Immunofluorescent imaging showed that $\mathrm{Ki}-67$ was significantly decreased in tumors administered with monotherapy as well as in those with combination therapies compared to that of control (Figure 6D). The combination of anti-PD-1 mAb with ManA at all tested doses significantly decreased MVD, demonstrating the anti-angiogenic effect of the combination therapy (Figure 6D). There was no reduction in MVD in tumors treated with different doses of anti-PD-1 mAb alone (Figure 6D).

In cancer, decreased pericyte coverage leads to leaky vessels as it regulates vessel permeability by supporting endothelial cells both mechanically and physically $[58,59]$. Thus, we investigated the effect of ManA on pericyte coverage of tumor vessels. Co-staining with anti-a-smooth muscles actin (a-SMA, a

Page 19/40 
marker for pericytes) and anti-CD31 showed the increase of a-SMA in ManA-treated groups compared to that in control and anti-PD-1 monotherapy groups (Figure S4F). This result suggests that the antiangiogenic effect of ManA is via improvement of pericyte coverage that ultimately reduces leaky vessels by normalizing it. We further performed TUNEL assay and measured apoptotic cells in both control and drug-treated tumor tissues. We found that apoptosis was markedly enhanced in combination therapy compared to monotherapy (Figure 6E). Taken together, these results showed that combination therapy of CMA activator and anti-PD-1 monoclonal antibody results in augmented anti-tumor activities.

\section{CMA activity and HIF-1 level are clinical prognostic markers in human lung cancer}

To assess the clinical efficacy of targeting HIF-1, we evaluated the correlation of HIF-1A expression in patient survival using the analytical tool, PREdiction of Clinical Outcomes from Genomic profiles (PRECOG) [60]. Based on Meta-Z scores, we found that expression of HIF1A and HSP90AA1 were positively correlated with poor clinical outcomes of lung cancer, whereas TFEB was reversely correlated (Figure 7A). In addition, we found that the expression of HIF1A mRNA in lung adenocarcinoma was reversely correlated with the expression of TFEB mRNA and positively related with the expression of HSP90AA1 (Figure 7B). Patient data further showed that patients with high HIF1A expression, as well as high HSP90AA1 and low TFEB expression, were associated with significantly poor survival in lung adenocarcinoma (Figure 7C). Importantly, survival rate was much higher in lung cancer patients with tumors with high TFEB and low HIF1A expression than the patients with tumors with low TFEB and high HIF1A expression (Figure 7D, left panel). Furthermore, survival rate was significantly higher in lung cancer patients with tumors with low HSP90AA1 and low HIF1A expression than the patients with tumors with high HSP90AA1 and high HIF1A expression (Figure 7D, right panel). These results support that ManA may have a high potency by targeting both HIF-1 and Hsp90 pathways in lung cancer patients.

This study shows that ManA inhibits Hsp90 chaperone activity by disrupting the interaction of Hsp90 with $\mathrm{F}_{1}$ Fo-ATP synthase. Inhibition of Hsp90 increases CMA activity through the interaction of CMA substrates and LAMP2A and increased nuclear localization of TFEB. These alterations induce CMAmediated HIF-1 a degradation, leading to suppression of glycolysis, immune tolerance, and angiogenesis. Therefore, CMA could effectively decrease tumor growth (Figure 7E).

\section{ADME properties of manassantin A}

Finally, we assessed ADME (absorption, distribution, metabolism, and excretion) properties of ManA in vivo/in vitro. The intravenous injection of ManA showed first-order elimination kinetics with $T \frac{1}{2}$ of $4.8 \pm$ $0.4 \mathrm{~h}$. Oral administration of ManA showed fast absorption with $\mathrm{T}_{\max }$ of $0.17 \mathrm{~h}$ and slow elimination profile with $T^{1} 1 / 2$ of $8.1 \pm 3.0 \mathrm{~h}$ (Figure S5 and Table S2). These $T^{1} / 2$ values were consistent with moderate clearance $(8.6 \pm 1.5 \mathrm{~mL} / \mathrm{min} / \mathrm{kg})$ of ManA and suggested its stability in the plasma and liver microsomes. Consistently, Table S3A illustrated its suitable plasma stability (i.e., $96.8 \pm 5.7 \%$ remaining after $2 \mathrm{~h}$ incubation) as well as metabolic stability (i.e., $103.4 \pm 3.8 \%$ remaining after $1 \mathrm{~h}$ incubation, half-life $>1 \mathrm{~h}$ ), respectively, in the plasma and liver microsomes from mice. ManA also showed good plasma stability 
with $95.0 \pm 3.5 \%$ and metabolic stability with $71.7 \pm 9.0 \%$ (Half-life of $2.1 \pm 0.1 \mathrm{~h}$ ) in human. In addition, fast $T_{\max }$ of ManA also consistent with the high absorptive permeability (i.e., Papp of $9.2 \pm 1.0 \times 10^{-6}$ $\mathrm{cm} / \mathrm{s}$ ) in absorptive direction when compared with the permeability of marker compounds such as caffeine, propranolol, ofloxacin, and atenolol (Table S3B). The permeability ratio of secretory direction to absorption direction (i.e., efflux ratio of 0.71 ) suggested limited involvement of efflux pumps in the intestine. Collectively, high permeability and limited involvement of efflux pumps expect favorable intestinal absorption of ManA following its oral administration, which is consistent with oral bioavailability of Man A (43.5\%) in this study.

\section{Discussion}

Autophagy has the capacity to degrade folded proteins, protein complexes and entire organelles. Various types of autophagy such as macroautophagy, microautophagy, and chaperone-mediated autophagy (CMA) co-exist in almost all mammalian cells. Macroautophagy and microautophagy are known to be non-selective degradation pathways for the organelle and various proteins, but CMA is a selective degradation pathway by targeting specific proteins having the pentapeptide motif KFERQ [61]. There has been increasing interest emphasizing that CMA activation can be a therapeutic strategy in the treatment of tumor [62]. However, it has not yet been reported how activation of CMA can inhibit hypoxic tumor growth. To investigate the role of CMA in tumor under hypoxia, we used ManA, which is a novel small molecule that mediates the potential crosstalk among CMA, HIF-1a, and Hsp90 of the hypoxic signaling pathway, as a mechanistic chemical probe.

The most intriguing question about HIF-1a degradation induced by ManA is how ManA activates CMA and reroutes HIF-1a degradation from proteasome to lysosome. Our CMAP analysis provides an important clue to this question. ManA functions as a Hsp90 inhibitor, and the inhibition of Hsp90 chaperone function decreased the stability of CMA substrates via CMA pathway. Importantly, unlike conventional Hsp90 inhibitors, ManA has no direct binding to Hsp90, but it indirectly modulates Hsp90 function by disrupting the Hsp90/ $\mathrm{F}_{1} \mathrm{~F}_{0}$-ATP synthase (chaperone/co-chaperone) interaction. Although conventional Hsp90 inhibitors have shown promising results in a limited number of disease settings, these inhibitors, in particular inhibitors of the N-terminal ATP-binding pocket, are known to induce the prosurvival heat shock response (HSR), retinopathy, gastrointestinal tract toxicity, and a range of doselimiting systemic toxicities [63]. They present serious challenges for clinical trials and hence there is an unmet need for the development of non-conventional Hsp90 inhibitors. In this regard, ManA is a promising lead compound for the development of Hsp90 modulators with a novel mechanism of action.

A few previous studies reported data that differed from those in our study. Some reported that CMA upregulates pro-oncogenic functions such as energy homeostasis and oncogenic cellular load and is thus required for oncogenic transformation and tumor growth $[53,64]$. On the other hand, a study showed that the overactivation of CMA suppresses tumor growth, metastasis, or cellular translation via the degradation of the M2 isoform of pyruvate kinase (PKM2), HKII, mutant p53, p65, and translation initiation factors such as EIF4A1, EIF4H, and DDX3X [4, 6, 65-67]. In addition, the CMA blockade 
contributes to malignant transformation by augmenting MYC oncogenic activity [5]. Specifically, under hypoxic conditions, CMA activation decreases HIF-1a and target genes encoding metabolic enzymes such as GLUT1and PDK1 in cancer cells and is correlated with the survival of neuronal cells $[8,68]$. These studies demonstrate that the anti-tumor effects of CMA are observed in hypoxic tumors but not in peritumoral areas characterized by active angiogenesis and hyperoxia or normoxia. Thus, our study suggests that inhibition of tumor growth was promoted by HIF-1a degradation through the overactivation of the CMA pathway. However, more extensive investigations are necessary to elucidate the effects of CMA on tumor growth via the alteration of the mechanistic pathway under normoxia and hypoxia.

Among multiple immune checkpoint inhibitors, PD-1/PD-L1 inhibitors have achieved success in clinical applications. Although therapeutic antibodies targeting PD-1/PD-L1 have been useful in understanding the mechanism of action of checkpoint inhibitors, they still have several limitations, including immunerelated adverse events, immunogenic response after repeated administrations, and poor tumor penetration. To address the disadvantages of antibody-based checkpoint inhibitors, there has been a growing interest in developing small molecule compounds in the past few years [69]. Our finding suggests that CMA-mediated HIF-1a degradation downregulates PD-L1 expression, and these changes are reversed by the overexpression of Hsp90, which increases $\mathrm{CD} 8^{+} \mathrm{T}$-cell infiltration. A previous study has revealed that CMA is required to maintain T-cell activation [70]. Although the study focused on $\mathrm{CD} 4^{+}$ $T$ cells, the role of $C M A$ in $C D 8^{+} T$ cells has not yet been identified $[70,71]$. Although our research did not clarify the function of CMA in $\mathrm{CD}^{+} \mathrm{T}$ cells, to the best of our knowledge, this is the first study showing that the overactivation of CMA in cancer cells plays a critical role in compromising the efficacy of the inhibitors of immune checkpoint inhibitors such as anti-PD-1.

In the 1970s, Folkman proposed the concept of targeting blood vessels in tumors to starve tumors of oxygen and nutrients. Since the overexpression of HIF-1a in tumors increases the expression of HIF target genes, such as VEGF, which are involved in tumor angiogenesis and chemoresistance [72, 73], targeting HIF-1a has multiple therapeutic benefits in inhibiting tumor growth. Consistent with previous studies showing that decreased pericyte coverage leads to leaky vessels, angiogenesis, and tumor cell dissemination in tumors [58,59], we observed that ManA-mediated CMA activation increases the pericyte coverage of tumor vessels and reduced hypoxic regions in tumors. This may be due to the alleviation of the deterioration of abnormal (e.g., leaky, chaotic) vascular structures caused by hypoxic stress [74]. However, other studies demonstrate that vascular pericytes in cancer support tumor vessels and facilitate premetastatic niche formation, metastasis, and tumor growth $[75,76]$. In addition, the upregulation of CMA in pericytes increases the immunosuppressive function of pericytes and tumor cell survival [77]. Keskin et al. differentiated the functions of pericytes under non-hypoxic and hypoxic conditions. Pericyte elimination in early-stage, non-hypoxic tumors inhibits angiogenesis, lung metastasis, and tumor growth; however, pericyte elimination in advanced-stage hypoxic tumor augments intra-tumor hypoxia, lung metastasis, and inhibits tumor growth [78]. Although the selective depletion of pericytes could effectively inhibit primary tumors exposed to hypoxia, the normalization of tumor vasculatures in order to increase drug perfusion and decrease hypoxic regions could be a more promising therapeutic approach to treating 
cancer than the inhibition of vessel density alone [79]. Anti-angiogenic therapy, such as anti-VEGF antibody (e.g., bevacizumab), usually results in the recurrence of hypoxia and induces angiogenesis again, thus increasing resistance against radiotherapy or chemotherapy [79]. Therefore, our study suggests that ManA enhances vascular normalization by improving pericyte coverage through the regulation of a master transcription factor HIF-1a, which inhibits angiogenesis and tumor growth.

\section{Conclusions}

The present study shows that Hsp90-mediated activation of CMA by ManA has potential anti-tumorigenic effects through targeting HIF-1a. CMA activation reroutes HIF-1 a degradation from the primary ubiquitination-proteasome pathway to the chaperon-mediated autophagy pathway. These findings set ManA distinct from other known HIF-1a inhibitors and CMA activators. Therefore, crosstalk among CMA, HIF-1a, and Hsp90 mediated by ManA will not only enhance our understanding of the hypoxia signaling pathway in tumor development and progression, but it will also provide opportunities for new and innovative therapeutics for cancer when immune checkpoint inhibitors are combined.

\section{Abbreviations}

CMA: Chaperon-mediated autophagy

ManA: Manassantin A

Hsp90: Heat shock protein 90

PD-1: Programmed death-1

TFEB: Transcription factor EB

Hsc70: Heat shock cognate protein of $70 \mathrm{kDa}$

HK2: Hexokinase 2

HIF-1: Hypoxia-inducible factor 1

PHDs: Proline hydroxylases

pVHL: Von Hippel Lindau protein

SAR: Structure-activity relationship

LLC: Lewis lung carcinoma

DMOG: Dimethyloxalylglycine

SAM: Significant Analysis of Microarrays 
CMAP: Connectivity Map

17-AAG: 17-(allylamino)-17-demethoxygeldanamycin

HS-10 resin: HS-10 immobilized on resin

TROSY: Transverse Relaxation-Optimized Spectroscopy

NMR: Nuclear Magnetic Resonance

SPR: Surface Plasma Resonance

Treg: Regulatory T cells

TIL: Tumor infiltrating lymphocytes

PRECOG: PREdiction of Clinical Outcomes from Genomic profiles

PKM2: M2 isoform of pyruvate kinase

\section{Declarations}

\section{Ethics approval and consent to participate}

Not applicable.

\section{Consent for publication}

Not applicable.

\section{Availability of data and materials}

All data generated or analyzed during this study are included in this article and its additional files.

\section{Competing interests}

The authors declare no competing financial interests.

\section{Funding}

This work was supported by the National Research Foundation of Korea (NRF-2017R1A2B3002227 and NRF-2020R1A5A2017323 to Y.M.L.) and bythe American Cancer Society (122057-RSG-12-045-01CDD to J.H.).

\section{Authors' Contributions}


J.-K.B., J.-H.J., S.H.L., M.-H. P. H.J. and N.B. conducted biochemical, cellular, and in vivo experiments and collected data. D.-Y.K. and T.N.S. synthesized manassantin A used in this study. S.J.P. and H.-H.K. conducted biophysical measurements. B.A.P. synthesized cruentaren A used in this study. E.J.M. and J.T.C. conducted gene expression profiling. D.H.W. and C.-T.L. conducted HS-10 resin experiments. E.J.M. conducted the analysis of patient survival data. Y.M.L., J.H., M.W.D., J.-T.C., and S.J.P. analyzed and discussed the data. Y.M.L. and J.H. conceived the project, designed the overall experimental strategy, wrote the manuscript with input from all the authors, and held overall responsibility for the study.

\section{Acknowledgements}

We are grateful to Dr. Timothy Haystead (Duke University) for HS-10, Masaaki Komatsu (Tokyo Metropolitan Institute of Medical Science) for ATG7 wild type and KO HeLa cells, Dr. Andrea Ballabio (Baylor College of Medicine) for TFEB-3 x Flag expression vector, Dr Brian S.J. Blagg (University of Notre Dame) for cruentaren A, and Dr. Leonard M. Neckers (National Cancer Institute) for pcDNA3- FLAG-tagged wild-type hHsp90a vectors used in this work. We thank Dr. Michael Fitzgerald (Duke University) and Dr. Robert Mook Jr. (Duke University) for helpful discussions.

\section{References}

1. Yang Z, Klionsky DJ. Eaten alive: a history of macroautophagy. Nat Cell Biol. 2010;12(9):814-22.

2. Tekirdag K, Cuervo AM. Chaperone-mediated autophagy and endosomal microautophagy: Joint by a chaperone. J Biol Chem. 2018;293(15):5414-24.

3. Ouchida AT, et al. Synergistic effect of a novel autophagy inhibitor and Quizartinib enhances cancer cell death. Cell Death Dis. 2018;9(2):138.

4. Vakifahmetoglu-Norberg $\mathrm{H}$, et al. Chaperone-mediated autophagy degrades mutant $\mathrm{p} 53$. Genes Dev. 2013;27(15):1718-30.

5. Gomes LR, Menck CFM, Cuervo AM. Chaperone-mediated autophagy prevents cellular transformation by regulating MYC proteasomal degradation. Autophagy. 2017;13(5):928-40.

6. Xia HG, et al. Degradation of HK2 by chaperone-mediated autophagy promotes metabolic catastrophe and cell death. J Cell Biol. 2015;210(5):705-16.

7. Schopf FH, Biebl MM, Buchner J. The HSP90 chaperone machinery. Nat Rev Mol Cell Biol. 2017;18(6):345-60.

8. Hubbi ME, et al. Chaperone-mediated autophagy targets hypoxia-inducible factor-1alpha (HIF1alpha) for lysosomal degradation. J Biol Chem. 2013;288(15):10703-14.

9. Semenza GL. Targeting HIF-1 for cancer therapy. Nat Rev Cancer. 2003;3(10):721-32.

10. Semenza GL. Hypoxia-Inducible Factors in Physiology and Medicine. Cell. 148(3): p. 399-408.

11. Semenza GL. Oxygen Sensing, Homeostasis, and Disease. N Engl J Med. 2011;365(6):537-47.

12. Ivan $\mathrm{M}$, et al. HIFalpha targeted for VHL-mediated destruction by proline hydroxylation: implications for 02 sensing. Science. 2001;292(5516):464-8. 
13. Vaupel P. Tumor microenvironmental physiology and its implications for radiation oncology. Semin Radiat Oncol. 2004;14(3):198-206.

14. Aebersold DM, et al. Expression of hypoxia-inducible factor-1alpha: a novel predictive and prognostic parameter in the radiotherapy of oropharyngeal cancer. Cancer Res. 2001;61(7):2911-6.

15. Moeller BJ, et al. Radiation activates HIF-1 to regulate vascular radiosensitivity in tumors: role of reoxygenation, free radicals, and stress granules. Cancer Cell. 2004;5(5):429-41.

16. Koukourakis MI, et al. Hypoxia-inducible factor (HIF1A and HIF2A), angiogenesis, and chemoradiotherapy outcome of squamous cell head-and-neck cancer. Int J Radiat Oncol Biol Phys. 2002;53(5):1192-202.

17. Schito L, Semenza GL. Hypoxia-Inducible Factors: Master Regulators of Cancer Progression. Trends Cancer. 2016;2(12):758-70.

18. Brizel DM, et al. Tumor hypoxia adversely affects the prognosis of carcinoma of the head and neck. Int J Radiat Oncol Biol Phys. 1997;38(2):285-9.

19. Hockel $\mathrm{M}$, et al. Association between tumor hypoxia and malignant progression in advanced cancer of the uterine cervix. Cancer Res. 1996;56(19):4509-15.

20. Moon EJ, et al. The potential role of intrinsic hypoxia markers as prognostic variables in cancer. Antioxid Redox Signal. 2007;9(8):1237-94.

21. Semenza GL. Evaluation of HIF-1 inhibitors as anticancer agents. Drug Discov Today. 2007;12(1920):853-9.

22. Wigerup C, Pahlman S, Bexell D. Therapeutic targeting of hypoxia and hypoxia-inducible factors in cancer. Pharmacol Ther. 2016;164:152-69.

23. Gaustad JV, et al. Vascular abnormalities and development of hypoxia in microscopic melanoma xenografts. J Transl Med. 2017;15(1):241.

24. Kwon DY, et al. Synthesis and Biological Evaluation of Manassantin Analogues for Hypoxia-Inducible Factor 1alpha Inhibition. J Med Chem. 2015;58(19):7659-71.

25. Kwak SH, et al. Evaluation of Manassantin A Tetrahydrofuran Core Region Analogues and Cooperative Therapeutic Effects with EGFR Inhibition. J Med Chem. 2020;63(13):6821-33.

26. $\mathrm{Kim} \mathrm{H}$, et al. Nucleophilic addition of organozinc reagents to 2-sulfonyl cyclic ethers: stereoselective synthesis of manassantins A and B. Org Lett. 2009;11(1):89-92.

27. Kusuma BR, Brandt GE, Blagg BS. Synthesis of cruentaren A. Org Lett. 2012;14(24):6242-5.

28. Dahia PL, et al. A HIF1alpha regulatory loop links hypoxia and mitochondrial signals in pheochromocytomas. PLoS Genet. 2005;1(1):72-80.

29. Hughes PF, et al. A highly selective Hsp90 affinity chromatography resin with a cleavable linker. Bioorg Med Chem. 2012;20(10):3298-305.

30. Park SJ, Kostic M, Dyson HJ. Dynamic Interaction of Hsp90 with Its Client Protein p53. J Mol Biol. 2011;411(1):158-73. 
31. Park SJ, et al. The client protein p53 adopts a molten globule-like state in the presence of Hsp90. Nat Struct Mol Biol. 2011;18(5):537-41.

32. Delaglio F, et al. NMRPipe: a multidimensional spectral processing system based on UNIX pipes. J Biomol NMR. 1995;6(3):277-93.

33. Johnson BA, Blevins RA. NMR View: A computer program for the visualization and analysis of NMR data. J Biomol NMR. 1994;4(5):603-14.

34. Gentles AJ, et al. The prognostic landscape of genes and infiltrating immune cells across human cancers. Nat Med. 2015;21(8):938-45.

35. Salceda S, Caro J. Hypoxia-inducible factor 1alpha (HIF-1alpha) protein is rapidly degraded by the ubiquitin-proteasome system under normoxic conditions. Its stabilization by hypoxia depends on redox-induced changes. J Biol Chem. 1997;272(36):22642-7.

36. Koga $\mathrm{H}$, et al. A photoconvertible fluorescent reporter to track chaperone-mediated autophagy. Nat Commun. 2011;2:386.

37. Seglen PO, Gordon PB. 3-Methyladenine: specific inhibitor of autophagic/lysosomal protein degradation in isolated rat hepatocytes. Proc Natl Acad Sci U S A. 1982;79(6):1889-92.

38. Hubbi ME, Semenza GL. Regulation of cell proliferation by hypoxia-inducible factors. Am J Physiol Cell Physiol. 2015;309(12):C775-82.

39. Frudd K, Burgoyne T, Burgoyne JR. Oxidation of Atg3 and Atg7 mediates inhibition of autophagy. Nat Commun. 2018;9(1):95.

40. Welsch T, et al. Eps8 is recruited to lysosomes and subjected to chaperone-mediated autophagy in cancer cells. Exp Cell Res. 2010;316(12):1914-24.

41. Kaushik S, Cuervo AM. The coming of age of chaperone-mediated autophagy. Nat Rev Mol Cell Biol. 2018;19(6):365-81.

42. Tusher VG, Tibshirani R, Chu G. Significance analysis of microarrays applied to the ionizing radiation response. Proc Natl Acad Sci U S A. 2001;98(9):5116-21.

43. Lamb J, et al. The Connectivity Map: using gene-expression signatures to connect small molecules, genes, and disease. Science. 2006;313(5795):1929-35.

44. Galam L, et al. High-throughput assay for the identification of Hsp90 inhibitors based on Hsp90dependent refolding of firefly luciferase. Bioorg Med Chem. 2007;15(5):1939-46.

45. Matts RL, Manjarrez JR. Assays for identification of Hsp90 inhibitors and biochemical methods for discriminating their mechanism of action. Curr Top Med Chem. 2009;9(15):1462-78.

46. Papathanassiu AE, et al. F1F0-ATP synthase functions as a co-chaperone of Hsp90-substrate protein complexes. Biochem Biophys Res Commun. 2006;345(1):419-29.

47. Hall JA, et al. Cruentaren A binds F1F0 ATP synthase to modulate the Hsp90 protein folding machinery. ACS Chem Biol. 2014;9(4):976-85.

48. Gradin $\mathrm{K}$, et al. Functional interference between hypoxia and dioxin signal transduction pathways: competition for recruitment of the Arnt transcription factor. Mol Cell Biol. 1996;16(10):5221-31. 
49. Palmieri $\mathrm{M}$, et al. mTORC1-independent TFEB activation via Akt inhibition promotes cellular clearance in neurodegenerative storage diseases. Nat Commun. 2017;8:14338.

50. Finn PF, et al. Effects of small molecules on chaperone-mediated autophagy. Autophagy. 2005;1(3):141-5.

51. Xue N, et al. Chaperone-mediated autophagy degradation of IGF-1Rbeta induced by NVP-AUY922 in pancreatic cancer. Cell Mol Life Sci. 2019;76(17):3433-47.

52. Shen S, et al. Cyclodepsipeptide toxin promotes the degradation of Hsp90 client proteins through chaperone-mediated autophagy. J Cell Biol. 2009;185(4):629-39.

53. Kon M, et al. Chaperone-mediated autophagy is required for tumor growth. Sci Transl Med. 2011;3(109):109ra117.

54. Andrade-Tomaz M, et al., The Role of Chaperone-Mediated Autophagy in Cell Cycle Control and Its Implications in Cancer. Cells, 2020. 9(9).

55. Noman MZ, et al. PD-L1 is a novel direct target of HIF-1alpha, and its blockade under hypoxia enhanced MDSC-mediated T cell activation. J Exp Med. 2014;211(5):781-90.

56. Barsoum IB, et al. A mechanism of hypoxia-mediated escape from adaptive immunity in cancer cells. Cancer Res. 2014;74(3):665-74.

57. Ruffell B, et al. Lymphocytes in cancer development: polarization towards pro-tumor immunity. Cytokine Growth Factor Rev. 2010;21(1):3-10.

58. Bergers G, Song S. The role of pericytes in blood-vessel formation and maintenance. Neuro Oncol. 2005;7(4):452-64.

59. Ribatti D, Nico B, Crivellato E. The role of pericytes in angiogenesis. Int J Dev Biol. 2011;55(3):261-8.

60 . Gentles AJ, et al. The prognostic landscape of genes and infiltrating immune cells across human cancers. Nat Med. 2015;21:938.

61. Bejarano E, Cuervo AM. Chaperone-mediated autophagy. Proc Am Thorac Soc. 2010;7(1):29-39.

62. Marinkovic M, et al., Autophagy Modulation in Cancer: Current Knowledge on Action and Therapy. Oxid Med Cell Longev, 2018. 2018: p. 8023821.

63. Garg G, Khandelwal A, Blagg BS. Anticancer Inhibitors of Hsp90 Function: Beyond the Usual Suspects. Adv Cancer Res. 2016;129:51-88.

64. Arias E, Cuervo AM. Pros and Cons of Chaperone-Mediated Autophagy in Cancer Biology. Trends Endocrinol Metab. 2020;31(1):53-66.

65. Lv L, et al. Acetylation targets the M2 isoform of pyruvate kinase for degradation through chaperonemediated autophagy and promotes tumor growth. Mol Cell. 2011;42(6):719-30.

66 . Tang J, et al. Impaired p65 degradation by decreased chaperone-mediated autophagy activity facilitates epithelial-to-mesenchymal transition. Oncogenesis. 2017;6(10):e387.

67. Hao Y, et al. Targetome analysis of chaperone-mediated autophagy in cancer cells. Autophagy. 2019;15(9):1558-71. 
68. Dohi E, et al. Hypoxic stress activates chaperone-mediated autophagy and modulates neuronal cell survival. Neurochem Int. 2012;60(4):431-42.

69. Ganesan A, et al. Comprehensive in vitro characterization of PD-L1 small molecule inhibitors. Sci Rep. 2019;9(1):12392.

70. Valdor $\mathrm{R}$, et al. Chaperone-mediated autophagy regulates $\mathrm{T}$ cell responses through targeted degradation of negative regulators of T cell activation. Nat Immunol. 2014;15(11):1046-54.

71. Molina ML, et al., Autophagy in the Immunosuppressive Perivascular Microenvironment of Glioblastoma. Cancers (Basel), 2019. 12(1).

72. Semenza GL. Intratumoral hypoxia, radiation resistance, and HIF-1. Cancer Cell. 2004;5(5):405-6.

73. Rankin EB, Giaccia AJ. Hypoxic control of metastasis. Science. 2016;352(6282):175-80.

74. Mazzone M, et al. Heterozygous deficiency of PHD2 restores tumor oxygenation and inhibits metastasis via endothelial normalization. Cell. 2009;136(5):839-51.

75. Cheng $L$, et al. Glioblastoma stem cells generate vascular pericytes to support vessel function and tumor growth. Cell. 2013;153(1):139-52.

76. Paiva AE, et al. Pericytes in the Premetastatic Niche. Cancer Res. 2018;78(11):2779-86.

77. Valdor R, et al. Glioblastoma ablates pericytes antitumor immune function through aberrant upregulation of chaperone-mediated autophagy. Proc Natl Acad Sci U S A. 2019;116(41):20655-65.

78. Keskin $D$, et al. Targeting vascular pericytes in hypoxic tumors increases lung metastasis via angiopoietin-2. Cell Rep. 2015;10(7):1066-81.

79. Jain RK. Antiangiogenesis strategies revisited: from starving tumors to alleviating hypoxia. Cancer Cell. 2014;26(5):605-22.

\section{Figures}


A

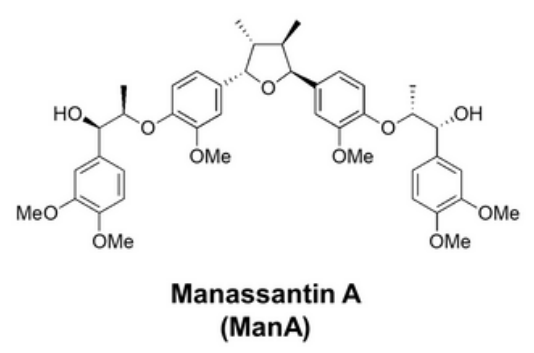

B

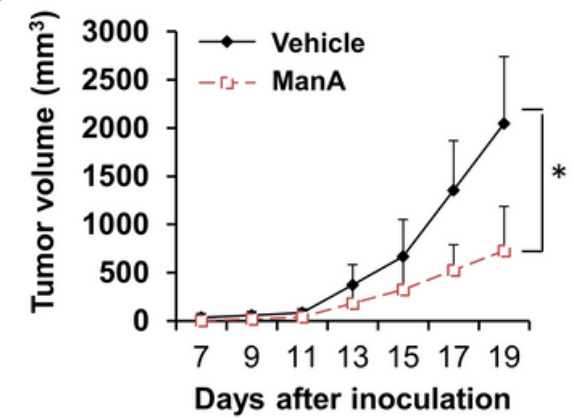

C

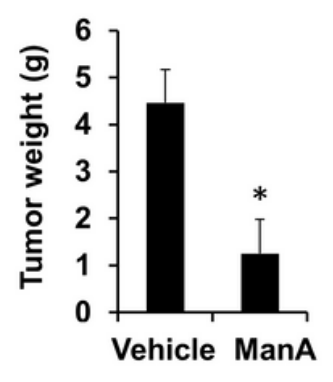

D
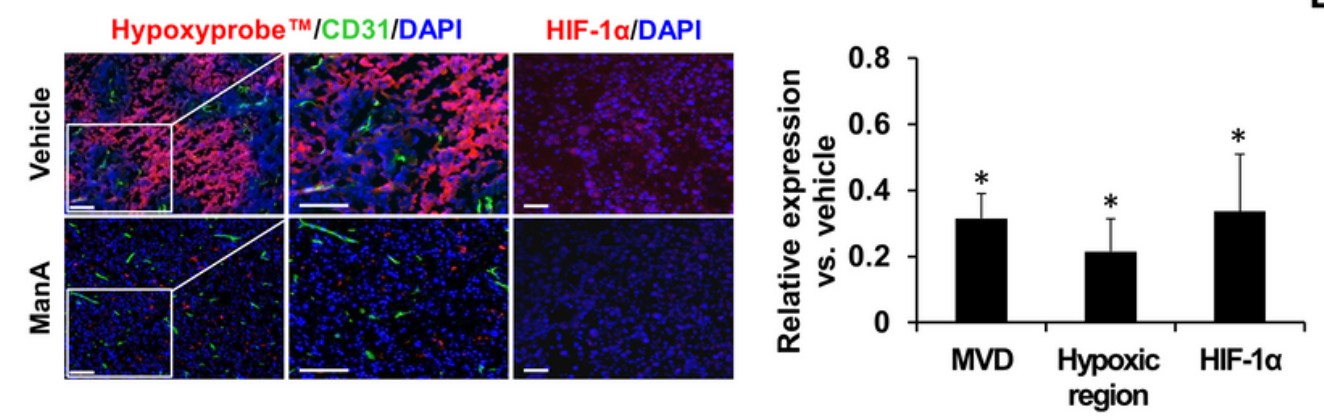

E

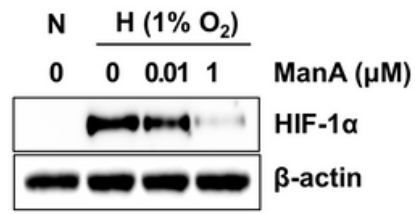

$\mathbf{F}$

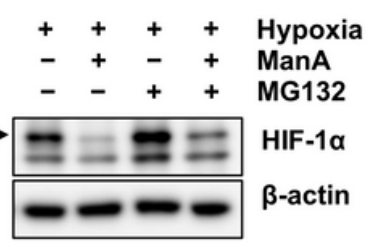

G

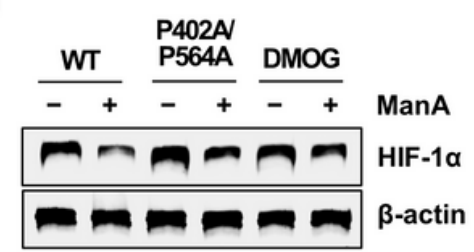

H

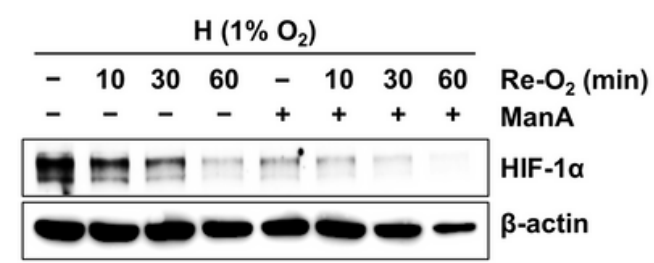

Figure 1

Manassantin A suppresses tumor growth by degrading HIF-1 a protein independent of the proteasomal degradation. (A) Structure of manassantin A (ManA). (B, C) Allograft tumor development in C57BL/6 mice inoculated with Lewis lung carcinoma (LLC) cells treated intraperitoneally with vehicle or ManA (5 $\mathrm{mg} / \mathrm{kg}$ ) every other day. Tumor volume (B) and tumor weight (C) were measured every other day and on the final day, respectively. ${ }^{*} p<0.05$ vs. Vehicle group. (D) Immunofluorescence of Hypoxyprobe ${ }^{\mathrm{TM}}$ (Pimo, red), CD31 (green), or HIF-1a (red) proteins in frozen sections of the tumors from (B) (Left). Mice were administered with pimonidazol $(60 \mathrm{mg} / \mathrm{kg}$ ) before sacrifice. Boxed areas are magnified at right. DAPI was used for nuclear staining. Scale bar $=100 \mu \mathrm{m}$. The positively stained area was image-analyzed for quantification (Right). ${ }^{*} \mathrm{p}<0.05$ vs. Vehicle group. (E) Western blot analysis of HIF-1a expression in HEK293T cells treated with ManA at indicated concentrations under hypoxic conditions for $24 \mathrm{~h}$. (F) Western blot analysis of HIF-1a expression in HEK293T cells treated with vehicle, ManA $(1 \mu \mathrm{M})$, or/and MG132 $(10 \mu \mathrm{M})$ under hypoxic conditions for $24 \mathrm{~h}$. Arrow indicates HIF-1a proteins. (G) Western blot analysis of HIF-1 a expression in HEK293T cells transfected with HIF-1 a wild type (WT) plasmid, P402A/P564A double mutant plasmid, or treated with DMOG $(20 \mu \mathrm{M})$ in the presence of vehicle or ManA $(1 \mu \mathrm{M})$ for $24 \mathrm{~h}$. $(\mathrm{H})$ Western blot analysis of HIF-1a expression in HEK293T cells treated with vehicle or ManA $(1 \mu \mathrm{M})$ under hypoxic conditions for $24 \mathrm{~h}$, followed by reoxygeneration for indicated times. 
A
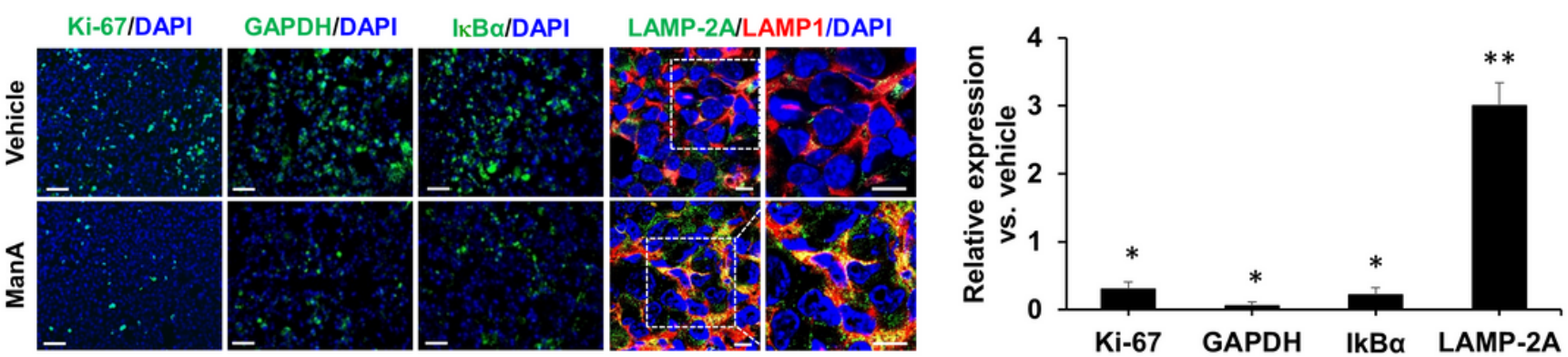

B
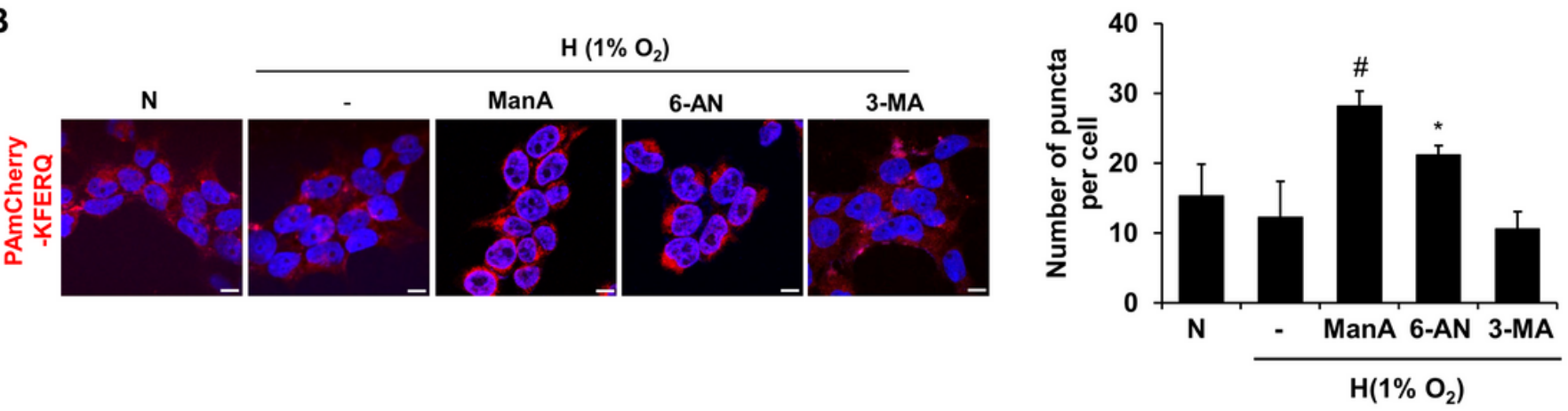

C

E

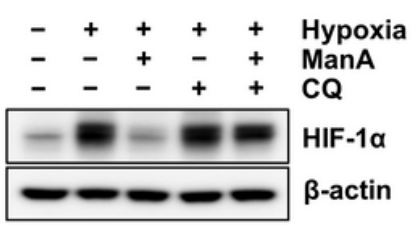

D

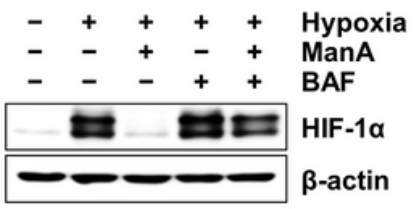

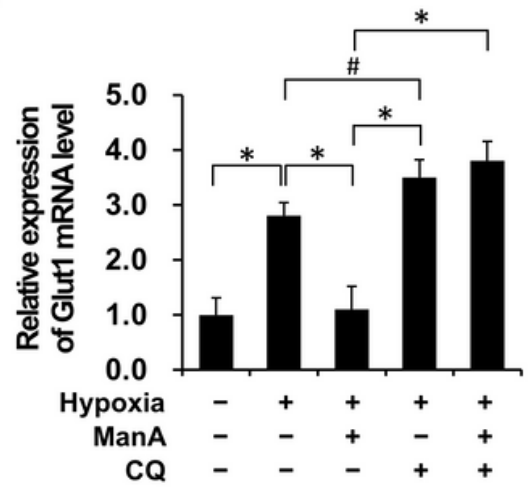

$\mathbf{F}$

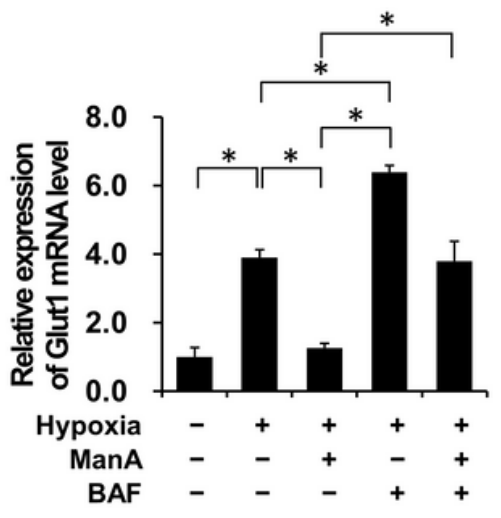

Figure 2

HIF-1 a protein is degraded by manassantin A through lysosome-mediated autophagy. (A) Immunofluorescence of Ki67 (green), GPADH (green), IKBa (green), and LAMP-2A (green)/LAMP1 (red) proteins in frozen sections of the tumors from Figure 1B (Left). DAPI was used for nuclear staining. Scale bar $=100 \mu \mathrm{m}(\mathrm{Ki}-67, \mathrm{GAPDH}, \mathrm{IKBa})$. Scale bar $=25 \mu \mathrm{m}$ (LAMP-2A). The positively stained area was image-analyzed for quantification (Right). ${ }^{*} \mathrm{p}<0.05 \mathrm{vs}$. Vehicle group. (B) Immunofluorescence of PAmCherry-KFERQ in photoactivated HEK293T cells treated with vehicle, ManA $(1 \mu \mathrm{M})$, 6-AN $(1 \mu \mathrm{M})$, or 3-MA (5 mM) under hypoxic conditions for $24 \mathrm{~h}$ (Left). Scale bar $=10 \mu \mathrm{m}$. CMA activity was analyzed as the number of fluorescent puncta per cell (Right). $\# p<0.01,{ }^{*} p<0.05$ vs. Vehicle group. (C) Western blot analysis of HIF-1 a expression in HEK293T cells treated with vehicle, chloroquine (CQ, $50 \mu \mathrm{M}$ ), and/or ManA $(1 \mu \mathrm{M})$ under hypoxic conditions for $8 \mathrm{~h}$. (D) Western blot analysis of HIF-1a expression in HEK293T cells treated with vehicle, bafilomycin (BAF, $10 \mathrm{nM})$, and/or ManA $(1 \mu \mathrm{M})$ under hypoxic conditions for 8 h. (E) qRT-PCR analysis of GLUT1 mRNA expression in HEK293T cells treated with 
vehicle, chloroquine (CQ, $50 \mu \mathrm{M})$, and/or ManA $(1 \mu \mathrm{M})$ under hypoxic conditions for 24 h. \#p < 0.01, * p < 0.05 between indicated two groups. (F) qRT-PCR analysis of GLUT1 mRNA expression in HEK293T cells treated with vehicle, bafilomycin (BAF, $10 \mathrm{nM})$, and/or ManA $(1 \mu \mathrm{M})$ under hypoxic conditions for $24 \mathrm{~h}$. ${ }^{*} \mathrm{p}$ $<0.01$ between indicated two groups.

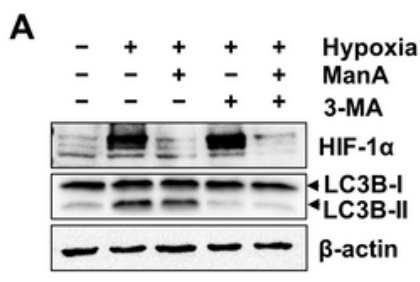

D

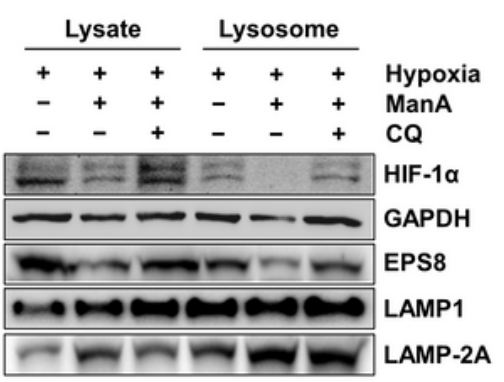

G

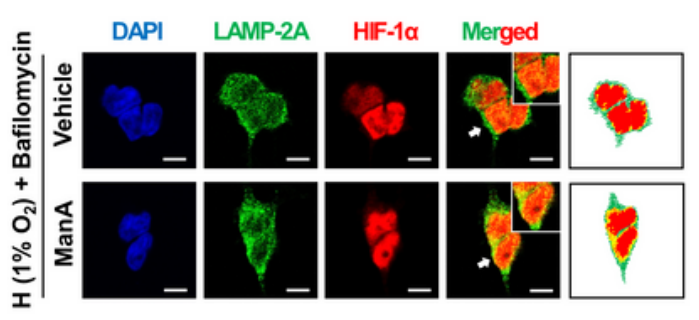

I

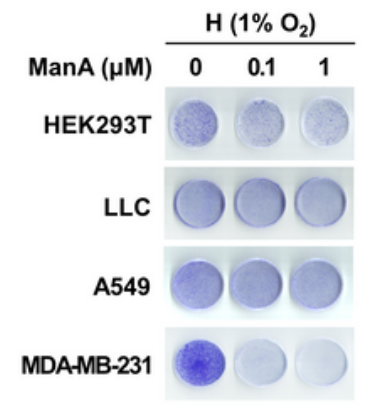

J

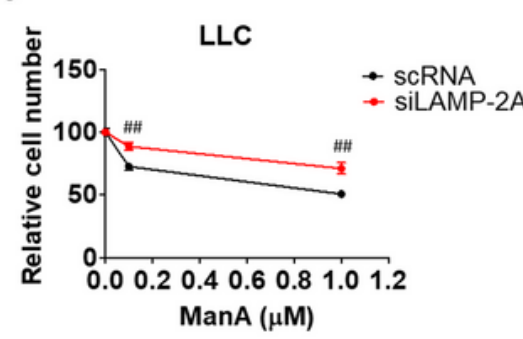

B

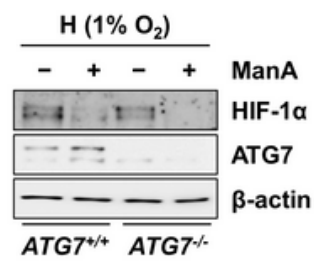

E

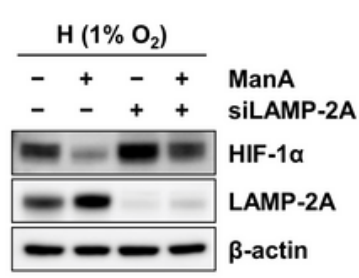

H

C

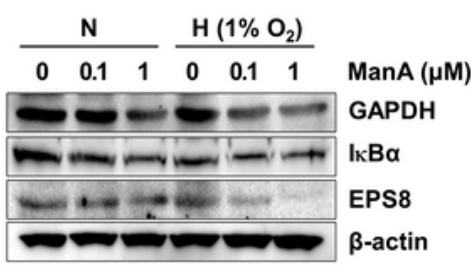

F
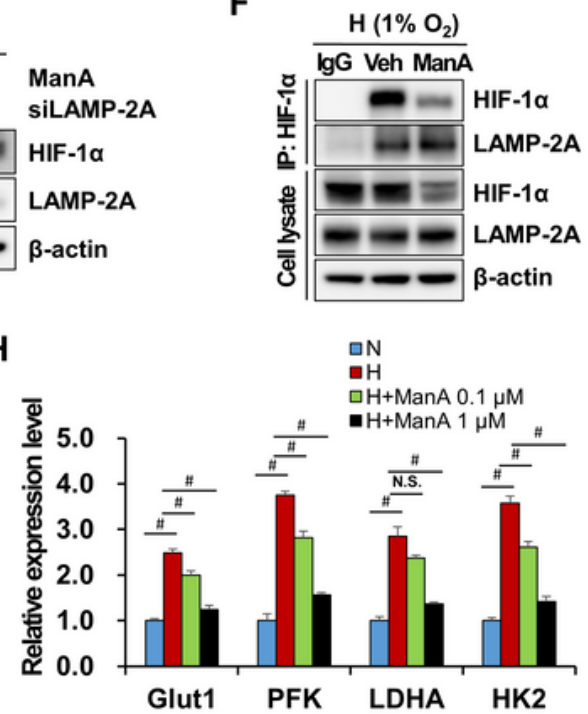

K

- HEK293T

= LLC

- A549

$=$ MDA-MB-231
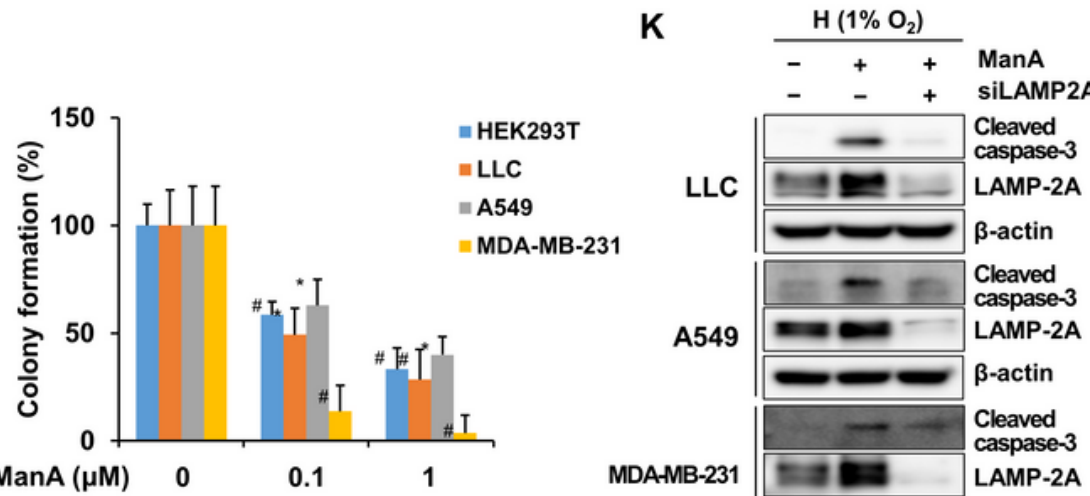

LLC

LC

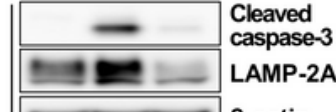

- $\beta$-actin

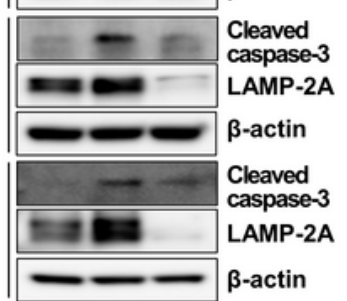

MDA-MB-231

MDA-MB-231
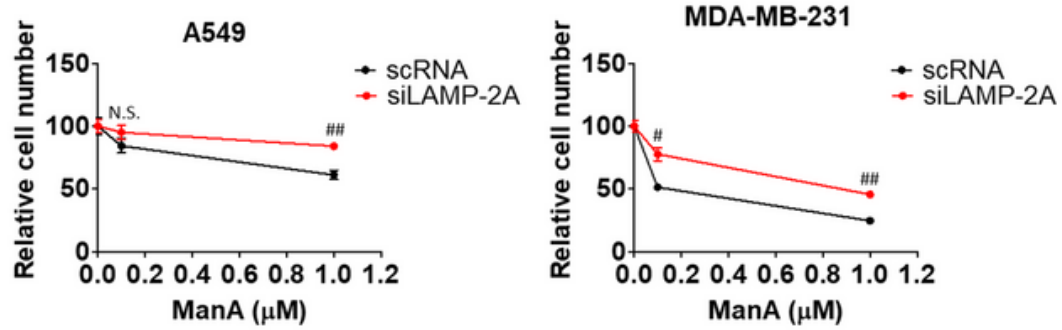

Figure 3 
Chaperone-mediated autophagy by manassantin A induces HIF-1 a degradation and inhibits cancer cell proliferation under hypoxic conditions. (A) Western blot analysis of HIF-1a and LC3B expression in HEK293T cells treated with 3-MA (5 mM) and/or ManA (100 nM) under hypoxic conditions for $8 \mathrm{~h}$. (B) Western blot analysis of HIF-1a and ATG7 expression in HeLa wild type or ATG7 knockout (KO) cells treated with vehicle or ManA $(1 \mu \mathrm{M})$ under hypoxic conditions for $24 \mathrm{~h}$. (C) Western blot analysis of GAPDH, EPS8, and IKBa in HEK293T cells treated with indicated concentrations of ManA under normoxia or hypoxic conditions for $24 \mathrm{~h}$. (D) Western blot analysis of HIF-1a, GAPDH, and EPS8 with whole-cell lysates and lysosomes of HEK293T cells treated with vehicle, chloroquine (CQ, $50 \mu \mathrm{M})$, and/or ManA (1 $\mu \mathrm{M})$ under hypoxic conditions for $8 \mathrm{~h}$. (E) Western blot analysis of HIF-1 a and LAMP-2A expression in HEK293T cells transfected with scrambled or LAMP-2A siRNA (siLAMP-2A) for $24 \mathrm{~h}$, followed by treatment with vehicle or ManA $(1 \mu \mathrm{M})$ under hypoxic conditions for $24 \mathrm{~h}$. $(\mathrm{F})$ Immunoprecipitation assay for HIF-1 a protein binding to LAMP-2A in HEK293T cells treated with vehicle or ManA (1 $\mu \mathrm{M})$ under hypoxic conditions for $8 \mathrm{~h}$. (G) Immunofluorescence of LAMP-2A (green) and HIF-1a (red) proteins in HEK293T cells treated with vehicle or ManA $(1 \mu \mathrm{M})$ in the presence of bafilomycin (BAF, $10 \mathrm{nM})$ under hypoxic conditions for $8 \mathrm{~h}(\mathrm{~F})$. DAPI was used for nuclear staining. Scale bar $=10 \mu \mathrm{m}$. Hand drawn images at the right panel show colocalization of LAMP-2A/HIF-1a (yellow). (H) qRT-PCR analysis of GLUT1, PFK, LDHA, and HK2 mRNA expression in HEK293T cells treated with indicated concentrations of ManA under hypoxic conditions for $24 \mathrm{~h}$. Data were normalized versus vehicle group and shown in graph below ( $n=3)$. N.S., not significant; \#p < 0.01 between indicated two groups. (I) Crystal violet staining of various cells treated with ManA at indicated concentrations under hypoxic conditions for 10 days. Quantitative graph is shown right panel. ${ }^{*} p<0.05, \# p<0.005$ vs vehicle control group. $(J)$ Cell proliferation of A549, LLC, or MDA-MB-231 cancer cells transfected with scrambled or LAMP-2A siRNA (siLAMP-2A) for $24 \mathrm{~h}$, followed by treatment with vehicle or ManA $(0.1,1 \mu \mathrm{M})$ under hypoxic conditions for 48 h. N.S., not significant, $\# p<0.01$, \#\#p < 0.001 vs. vehicle control group. (K) Western blot analysis of cleaved caspase-3 expression of A549, LLC, or MDA-MB-231 cells transfected with scrambled or LAMP-2A siRNA (siLAMP-2A) for $24 \mathrm{~h}$, followed by treatment with vehicle or ManA $(1 \mu \mathrm{M})$ under hypoxic conditions for $48 \mathrm{~h}$. 
A

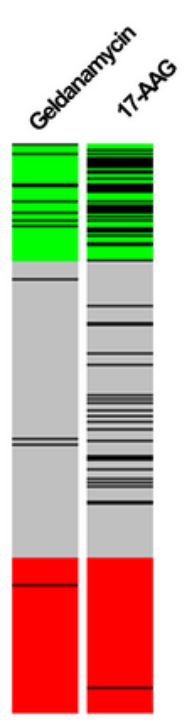

F

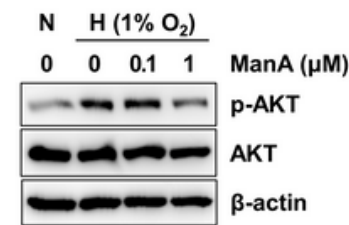

H

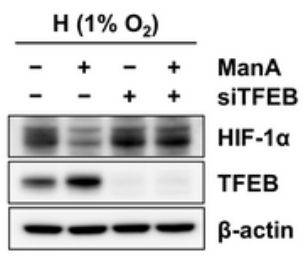

J

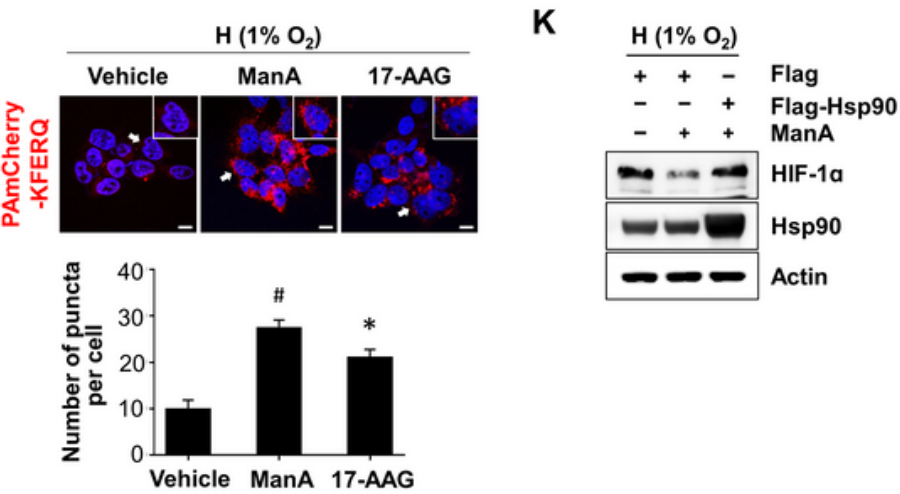

B

D

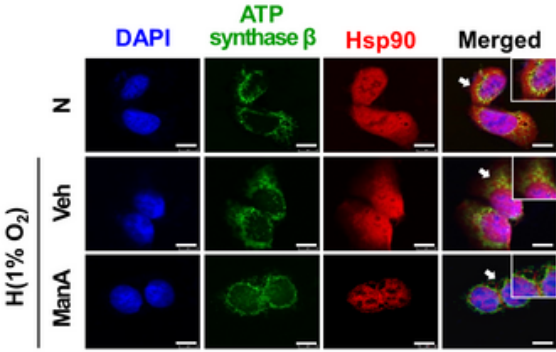

G

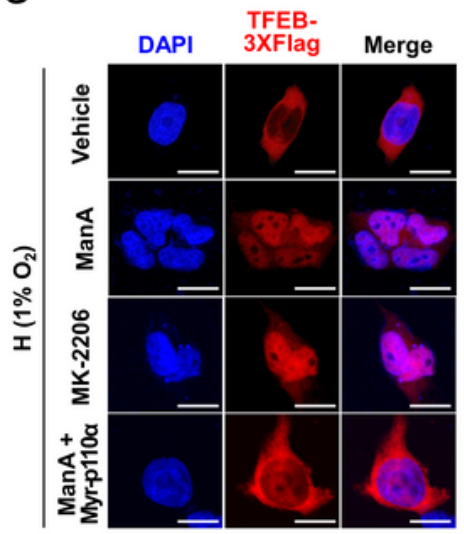

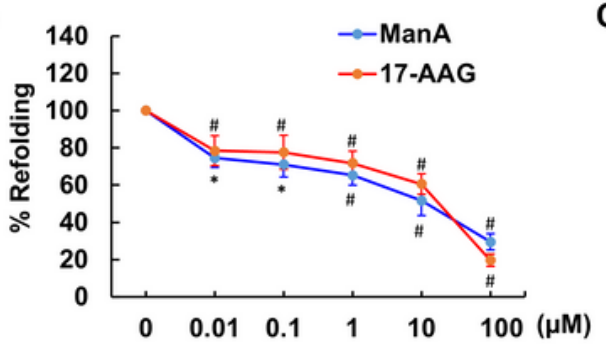

E

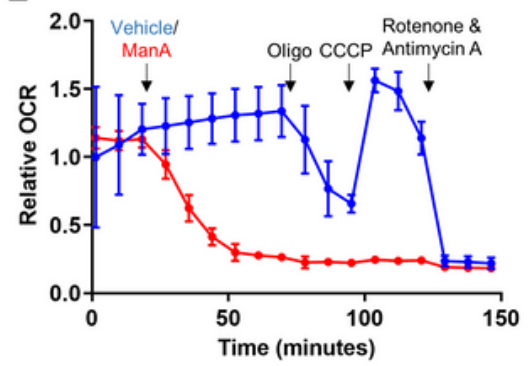

I

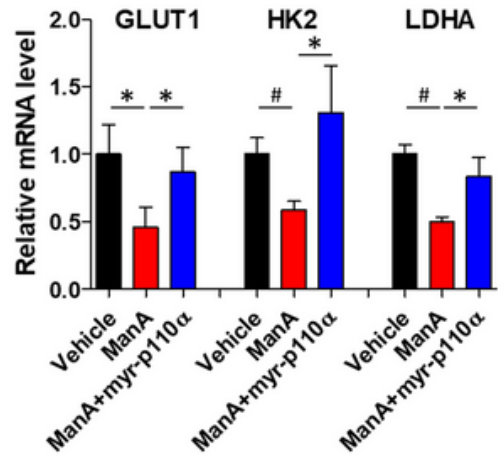

L

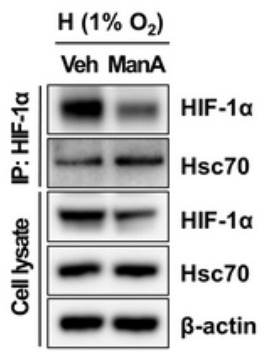

Figure 4

Chaperone-mediated autophagy by manassantin A is associated with Hsp90 chaperon function. (A) Connectivity map analysis of ManA-induced transcriptional changes compared with geldanamycin and 17-AAG, two different Hsp90 inhibitors. (B) Luciferase refolding assay in HEK293T cells treated with ManA or 17-AAG at indicated concentrations. Refolding percentage was calculated relative to vehicle group for $100 \%$. *p < 0.05, \#p < 0.01 vs. vehicle group. (C) Immunoprecipitation assay for ATP synthase $\beta$ 
protein binding to HSP90 in HEK293T cells treated with vehicle, ManA, or MA02 (an inactive ManA analog) at indicated concentrations under hypoxic conditions for $24 \mathrm{~h}$. (D) Immunofluorescence of ATP synthase $\beta$ and HSP90 proteins in HEK293T cells treated with vehicle or ManA $(1 \mu \mathrm{M})$ under hypoxic conditions for $24 \mathrm{~h}$. DAPI was used for nuclear staining. Scale bar $=10 \mu \mathrm{m}$. (E) The oxygen consumption rate (OCR) of HEK293T at the indicated time points after ManA $(1 \mu \mathrm{M})$ treatment. (F) Western blot analysis of p-AKT expression in HEK293T cells treated with ManA at indicated concentrations under hypoxic conditions for $24 \mathrm{~h}$. (G) Immunofluorescence of TFEB (red) in HEK293T cells expressing TFEB-3 $X$ Flag transfected with control or Myr-p110a expression vector for $24 \mathrm{~h}$, followed by treatment with ManA $(1 \mu \mathrm{M})$ or MK-2206 $(1 \mu \mathrm{M})$ under hypoxic condition for $24 \mathrm{~h}$. DAPI was used for nuclear staining. Scale bar $=10 \mu \mathrm{m} .(\mathrm{H})$ Western blot analysis of HIF-1 $\mathrm{a}$ and TFEB expression in HEK293T cells transfected with scrambled or TFEB siRNA (siTFEB) for $24 \mathrm{~h}$, followed by treatment with vehicle or ManA $(1 \mu \mathrm{M})$ under hypoxic conditions for $24 \mathrm{~h}$. (I) qRT-PCR analysis of GLUT1, HK2, and LDHA mRNA expression in HEK293T cells transfected with Myr-p110a expression vector or empty vector for $24 \mathrm{~h}$, followed by treatment with ManA $(1 \mu \mathrm{M})$ under hypoxic condition for $24 \mathrm{~h}$. Data were normalized versus vehicle control group $(n=3) .{ }^{*} p<0.05, \# p<0.01$ between indicated two groups. (J) Immunofluorescence of PAmCherry-KFERQ in photoactivated HEK293T cells treated with vehicle, ManA $(1 \mu \mathrm{M})$, or 17-AAG $(1 \mu \mathrm{M})$ under hypoxic conditions for $24 \mathrm{~h}$ (Upper). Scale bar $=10 \mu \mathrm{m}$. CMA activity was analyzed as the number of fluorescent puncta per cell (Lower). $\# p<0.01,{ }^{\star} p<0.05 \mathrm{vs}$. Vehicle group. (K) Western blot analysis of HIF-1a expression in HEP293T cells transfected with control or Flag-Hsp90a expression vector for $48 \mathrm{~h}$, followed by treatment with vehicle or ManA $(1 \mu \mathrm{M})$ under hypoxic condition for $24 \mathrm{~h}$. (L) Immunoprecipitation assay for HIF-1a protein binding to Hsc70 in HEK293T cells treated with vehicle or ManA $(1 \mu \mathrm{M})$ under hypoxic conditions for $8 \mathrm{~h}$. 
A

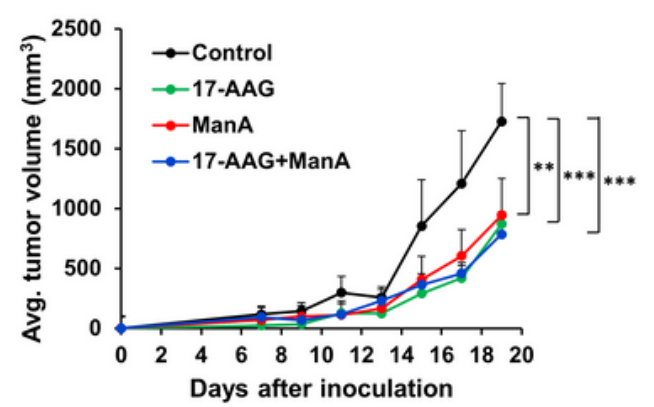

B

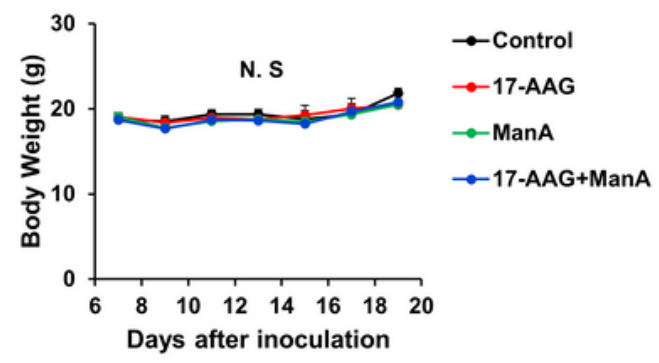

C
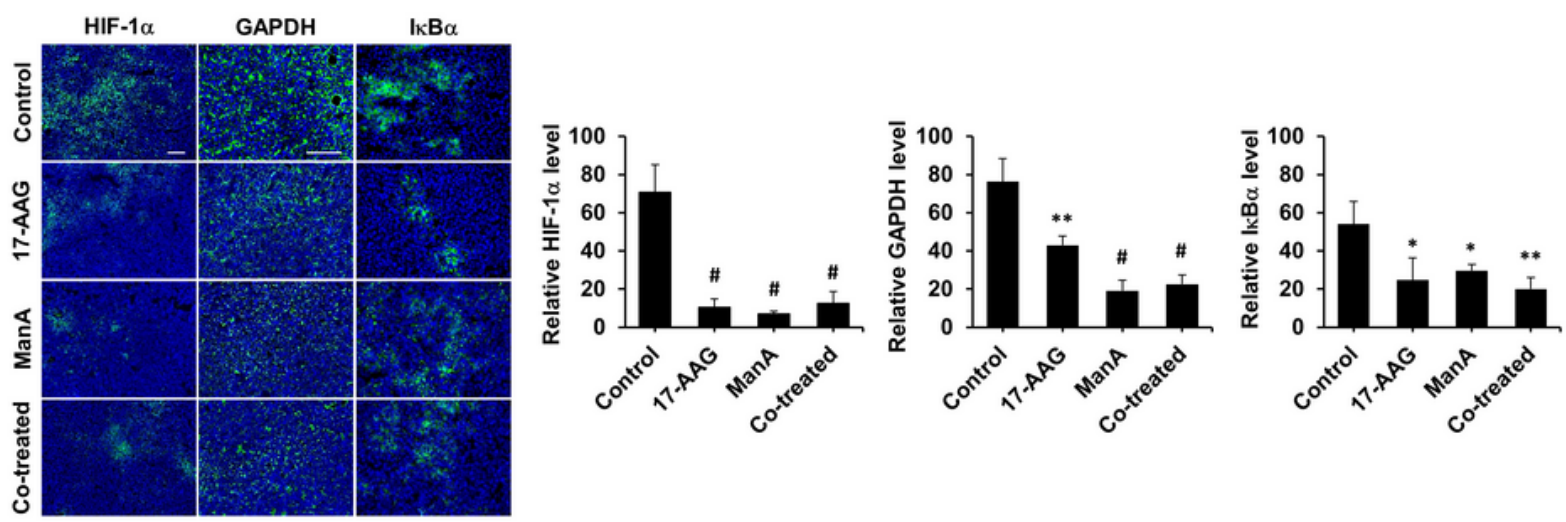

D

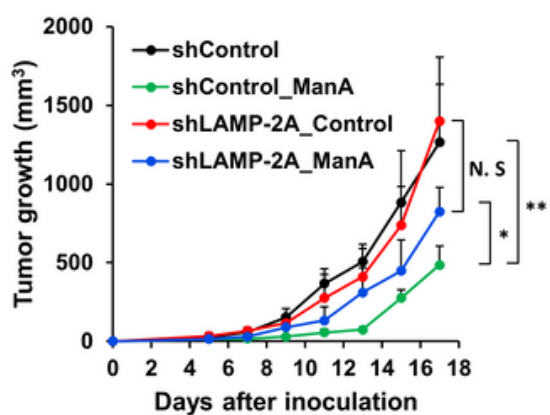

E

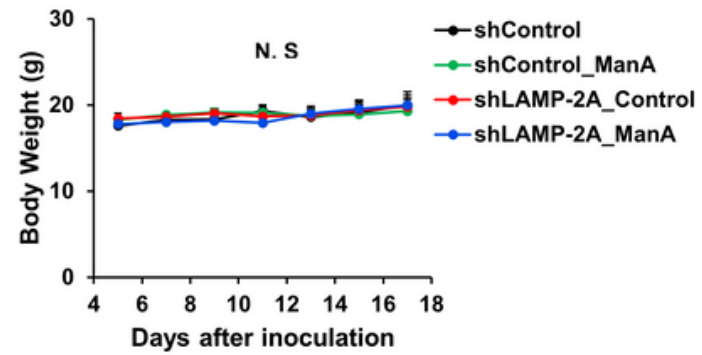

F
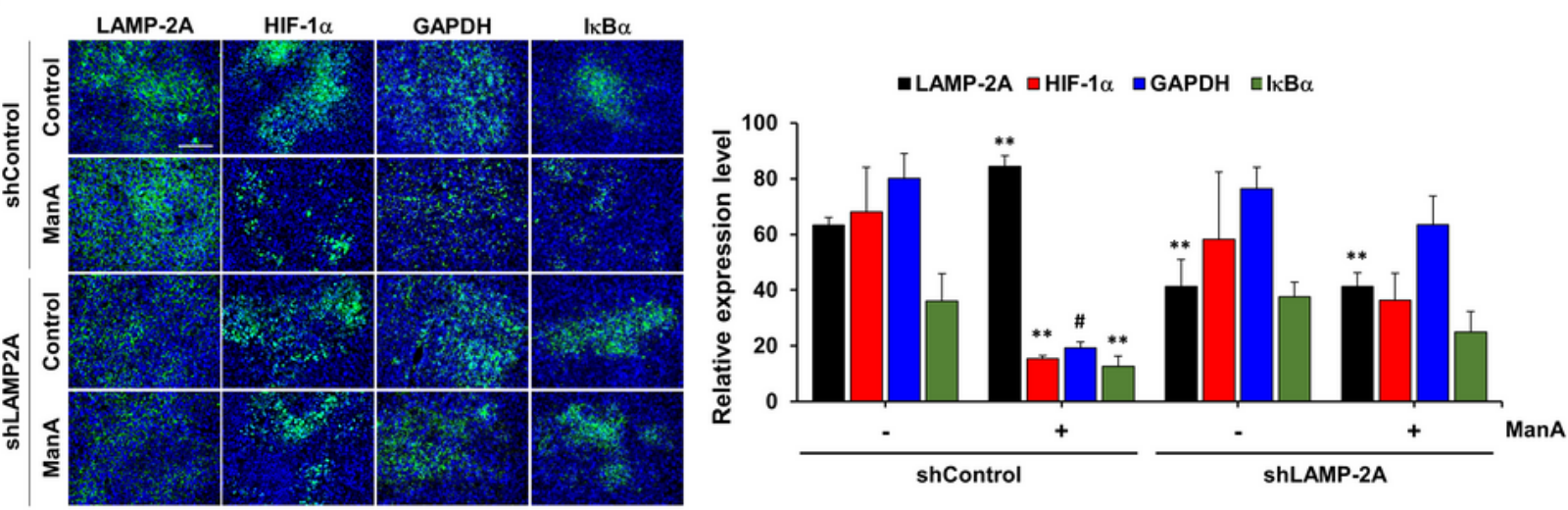

Figure 5

Inhibition of Hsp90 or LAMP-2A do not augment the effect of anti-tumor growth by ManA. (A and B) LLC cells were inoculated in the flank of C57BL/6 mice. ManA (2 mg/kg) and/or 17-AAG (25 mg/kg) were injected intraperitoneally every other day ( $n=6 /$ group). Tumor volume $(A)$ and body weight (B) were measured every other day. N.S., not significant; $\star \star ~ p<0.01, \star \star \star P<0.001$ vs. Vehicle control. (C) Immunofluorescence of HIF-1a (green), GPADH (green), and IKBa (green) in frozen sections of the tumors 
from (A). DAPI was used for nuclear staining. Scale bar $=50 \mu \mathrm{m}$ (Left). The positively stained area was image-analyzed for quantification (Right). ${ }^{*} p<0.05,{ }^{*} p<0.01, \# p<0.0001$ vs. Vehicle group. (D and E) LLC cells expressing shControl or shLAMP-2A were inoculated in the flank of C57BL/ 6 mice. ManA ( $5 \mathrm{mg} / \mathrm{kg})$ and/or vehicle were injected intraperitoneally every other day ( $n=5 /$ group). Tumor volume (D) and body weight (E) were measured every other day. N.S., not significant; ${ }^{*} p<0.05,{ }^{*} p<0.01$ vs. Vehicle control. (F) Immunofluorescence of LAMP-2A (green), HIF-1a (green), GPADH (green), and IKBa (green) in frozen sections of the tumors from (D). DAPI was used for nuclear staining. Scale bar $=50 \mu \mathrm{m}$ (Left). The positively stained area was image-analyzed for quantification (Right). ${ }^{\star \star} p<0.01, \# p<0.0001$ vs. Vehicle group. 
A

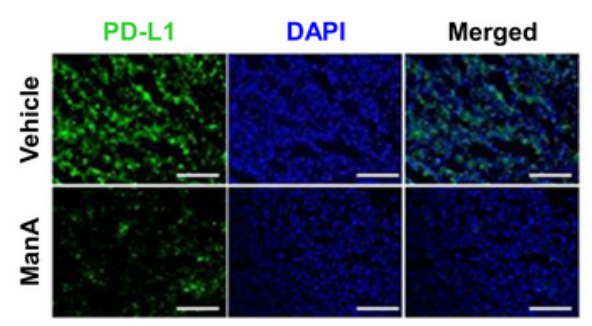

B

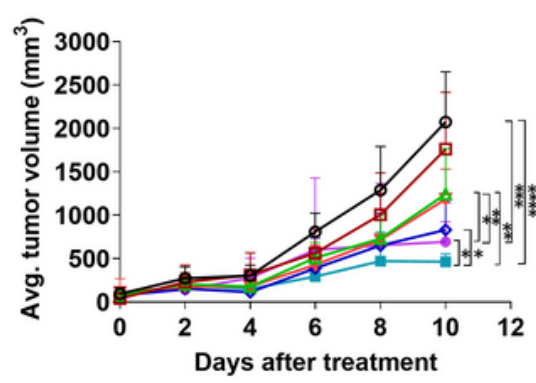

- Untreated Control

$\because$ Vehicle Control

$\triangle \operatorname{ManA}(2 \mathrm{mg} / \mathrm{kg})$

7 antiPD-1 Ab (5 mg/kg)

- antiPD-1 Ab (15 mg/kg)

- ManA (2 mg/kg) + antiPD-1 Ab (5 mg/kg)

- ManA (2 mg/kg) + anti-PD-1 Ab (15 mg/kg)

C
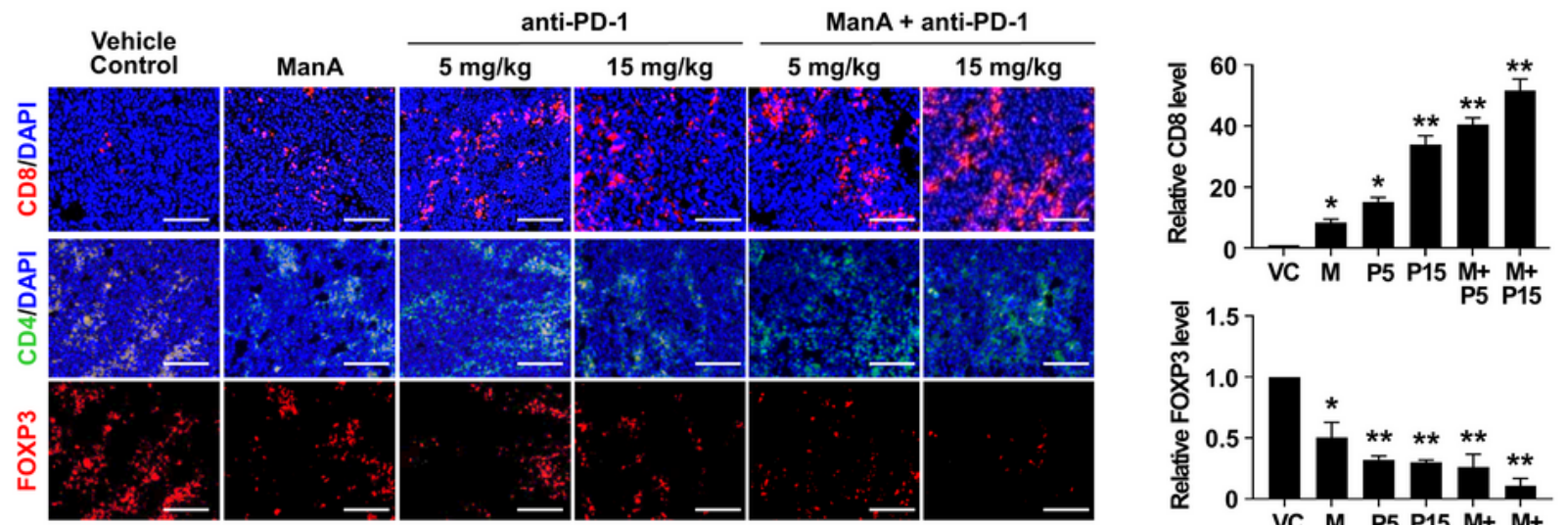

D
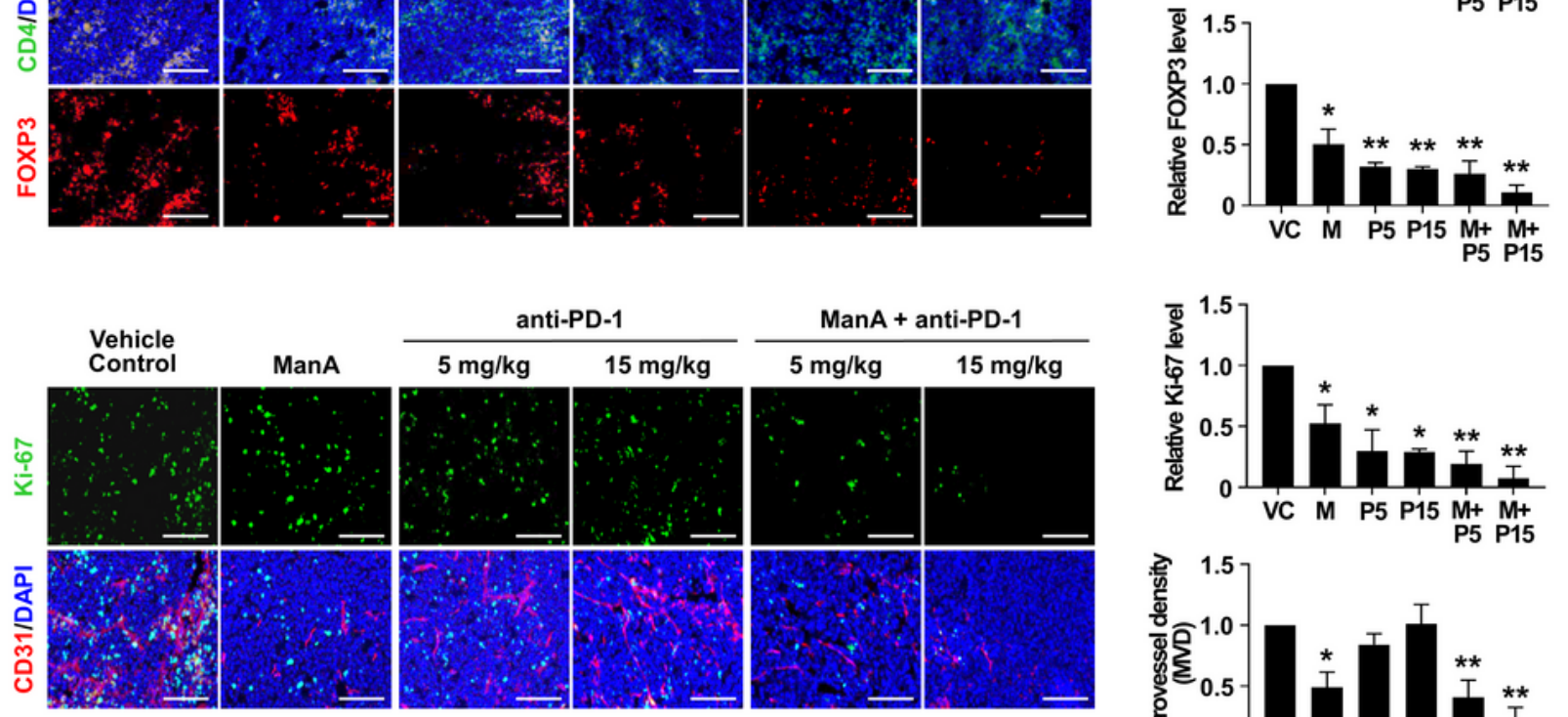

E
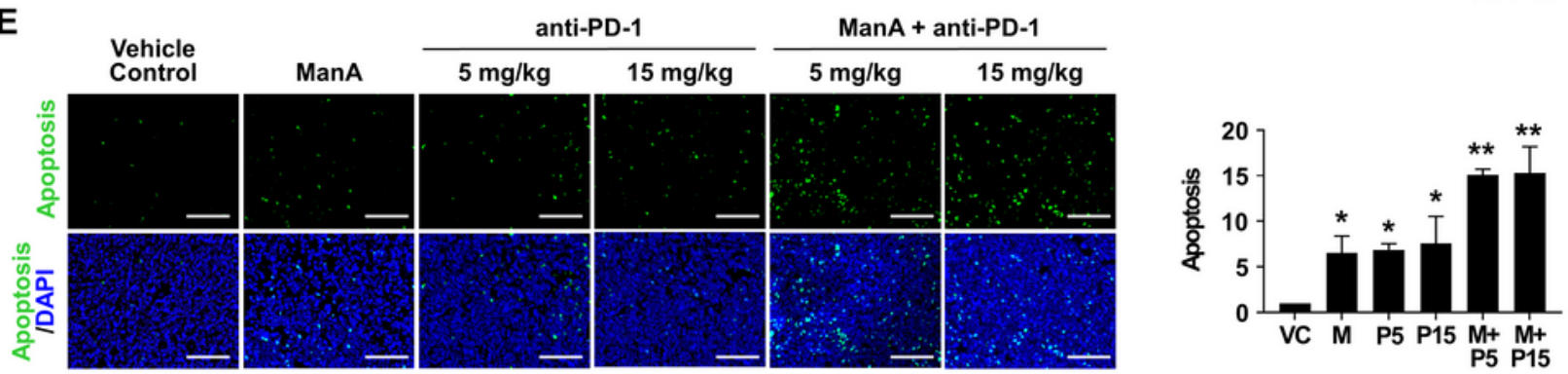

\section{Figure 6}

Activation of chaperone-mediated autophagy by manassantin A shows additive or cooperative antitumor effects in combination with anti-PD-1 antibody. (A) Immunofluorescence of PD-L1 protein in frozen tumor tissue sections from Figure 1B. DAPI was used for nuclear staining. Scale bar = $100 \mu \mathrm{m}$. (B) Allograft tumor development in C57BL/6 mice inoculated with Lewis lung carcinoma (LLC) cells treated intraperitoneally with anti-mouse PD-1 (5, 15 mg/kg) and/or ManA (2 mg/kg) (n = 4 per group) every 
other day. Tumor volume was measured every other day. (C) Immunofluorescence of CD8, CD4, and FOXP3 proteins in frozen tumor tissue sections from Figure 5B (Left). DAPI was used for nuclear staining. (D) Immunofluorescence of CD31 and Ki-67 proteins in frozen tumor tissue sections from Figure 5B (Left). DAPI was used for nuclear staining. (E) TUNEL assay of frozen tumor tissue sections from Figure 5B (Left). DAPI was used for nuclear staining. Scale bar $(C-E)=50 \mu \mathrm{m}$. The positively stained area was

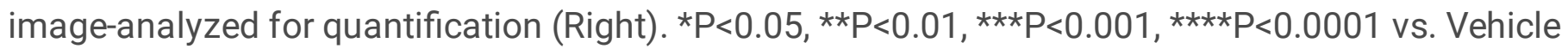
control. VC, vehicle control; M, ManA (2 mg/kg); P5, Anti-PD-1 (5 mg/kg); P15, anti-PD-1 (15 mg/kg).

A

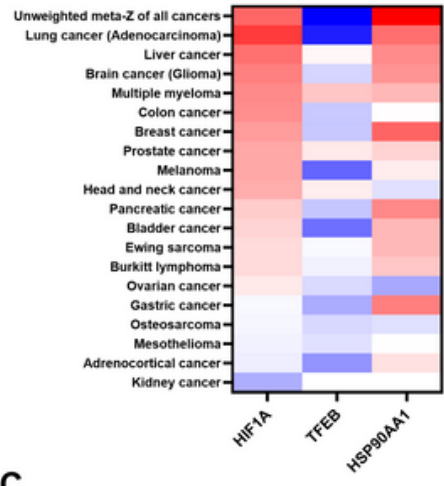

C
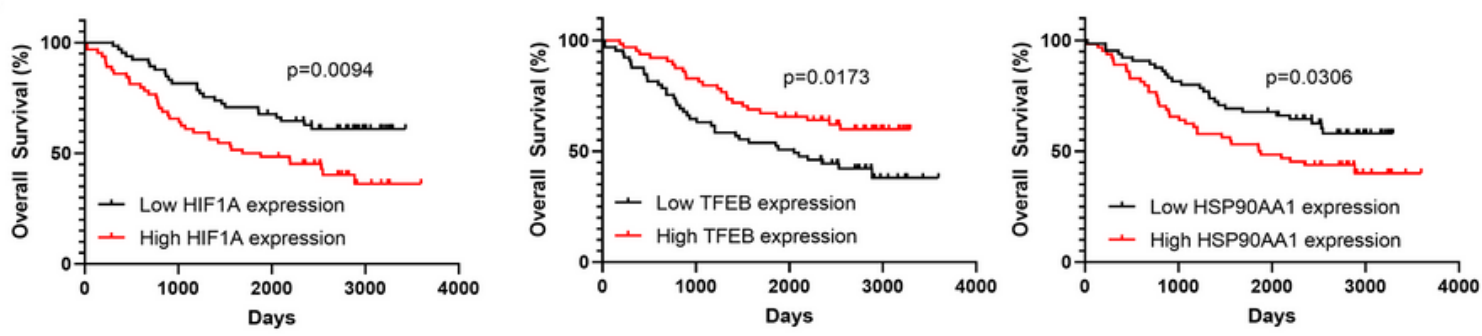

B

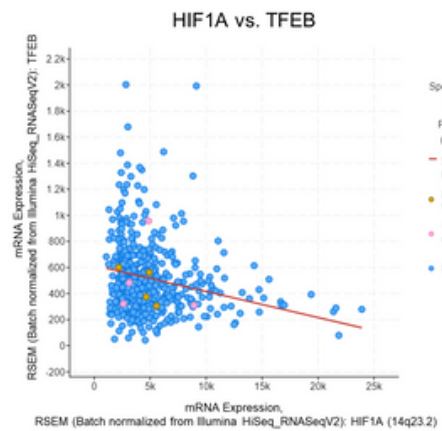

D

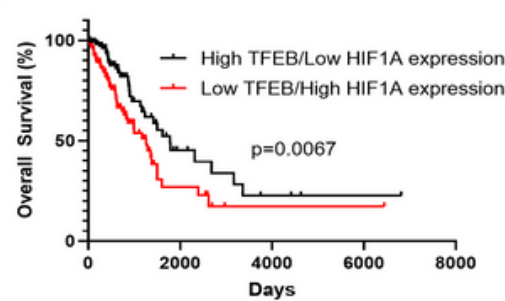

(Low TFEB/High HIF1A $n=137$, High TFEB/Low HIF1A $n=129$ )

E

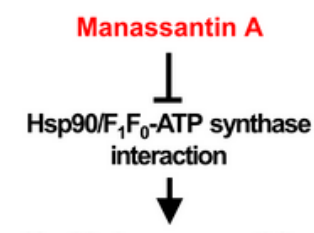

Hsp90 chaperone activity

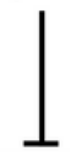

Chaperone mediated autophagy (CMA)
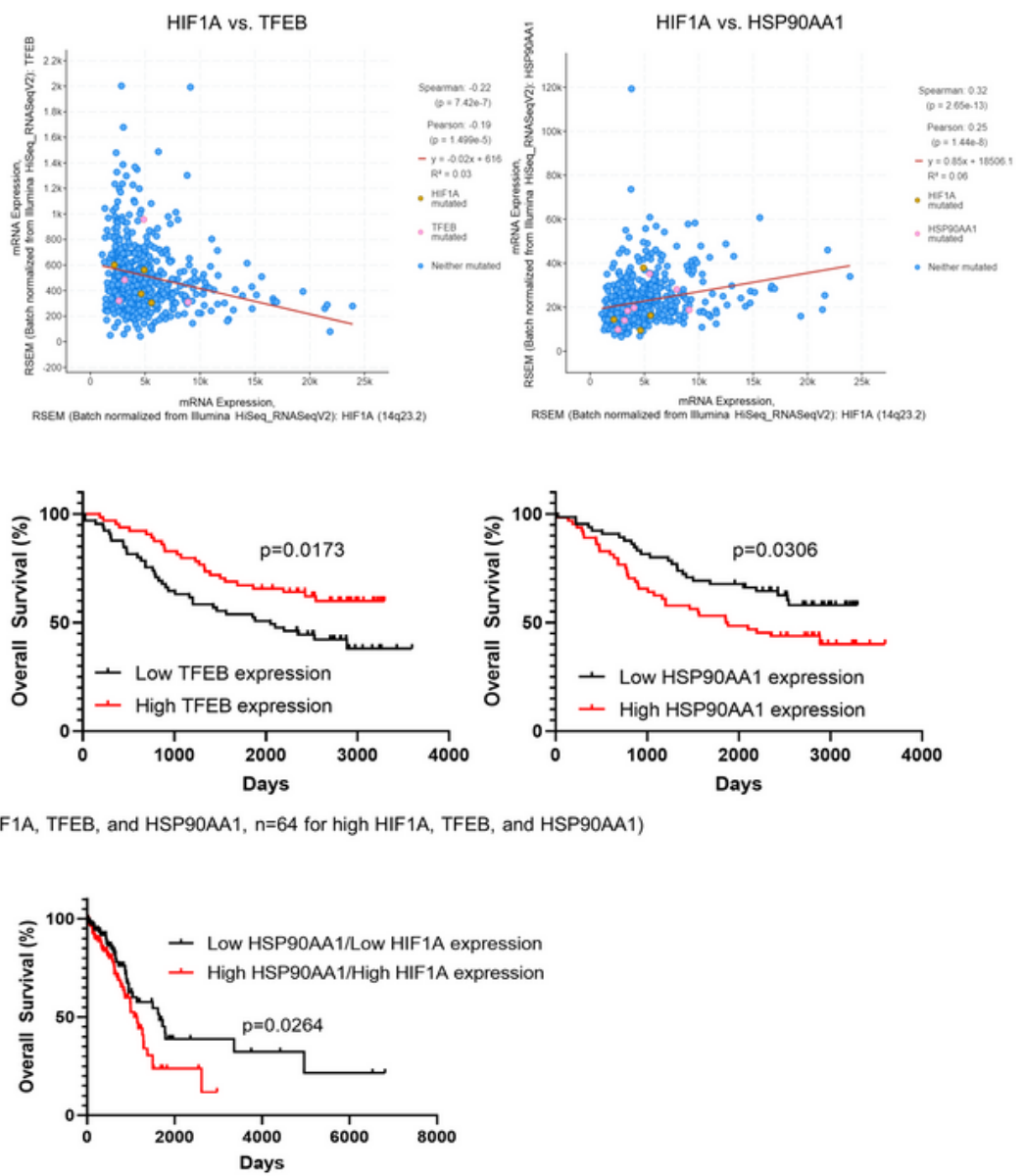

(Low HSP90AA1/Low HIF1A $n=145$, High HSP90AA1/High HIF1A $n=145$ )
AKT phosphorylation

TFEB nuclear localization 


\section{Figure 7}

Expression of HIF1A and HSP90 predicts overall survival in lung and breast cancer patients. (A) Combined $Z$ scores from each study indicated that HIF1A, TFEB, and HSP90AA1 expression was most highly correlated with lung cancer. (B) The correlations between HIF1A and TFEB (left), or between HIF1A and HSP90AA1 (right), expression by RSEM in lung cancer patient. (C) Correlation of HIF1A (Left, $p=$ 0.0094), TFEB (Middle, $p=0.0173$ ), and HSP90AA1 (Right, $p=0.0306$ ) expression with overall survival in lung cancer patient. (D) Correlation of TFEB/HIF1A (Left, $p=0.0067$ ), and HSP90AA1/HIF1A (Right, $p=$ 0.0264 ) expression with overall survival in lung cancer patient. (E) Schematic representation of molecular mechanism underlying antitumor activity of ManA.

\section{Supplementary Files}

This is a list of supplementary files associated with this preprint. Click to download.

- SupplementaryMaterial.docx 